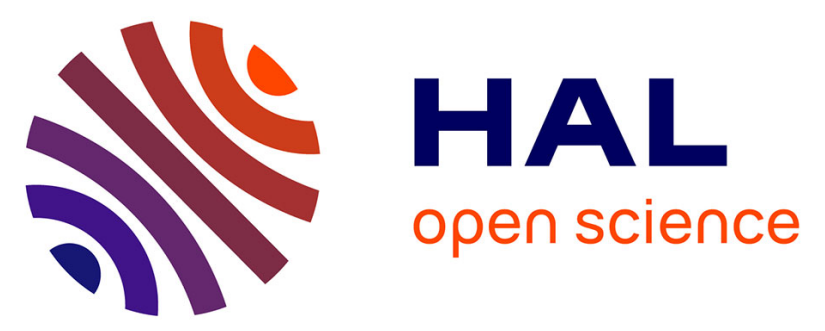

\title{
Controlled Anchoring of Iron Oxide Nanoparticles on Polymeric Nanofibers: Easy Access to Core@Shell Organic-Inorganic Nanocomposites for Magneto-Scaffolds
}

Hussein Awada, Assala Al Samad, Danielle Laurencin, Ryan Gilbert, Xavier Dumail, Ayman El Jundi, Audrey Bethry, Rebecca Pomrenke, Christopher Johnson, Laurent Lemaire, et al.

\section{- To cite this version:}

Hussein Awada, Assala Al Samad, Danielle Laurencin, Ryan Gilbert, Xavier Dumail, et al.. Controlled Anchoring of Iron Oxide Nanoparticles on Polymeric Nanofibers: Easy Access to Core@Shell OrganicInorganic Nanocomposites for Magneto-Scaffolds. ACS Applied Materials \& Interfaces, 2019, 11 (9), pp.9519-9529. 10.1021/acsami.8b19099 . hal-02347893

\section{HAL Id: hal-02347893 \\ https://hal.science/hal-02347893}

Submitted on 25 Aug 2021

HAL is a multi-disciplinary open access archive for the deposit and dissemination of scientific research documents, whether they are published or not. The documents may come from teaching and research institutions in France or abroad, or from public or private research centers.
L'archive ouverte pluridisciplinaire $\mathbf{H A L}$, est destinée au dépôt et à la diffusion de documents scientifiques de niveau recherche, publiés ou non, émanant des établissements d'enseignement et de recherche français ou étrangers, des laboratoires publics ou privés. 


\title{
Controlled Anchoring of Iron-Oxide Nanoparticles
}

\author{
on Polymeric Nanofibers: Easy Access to
}

Core@Shell Organic-Inorganic Nanocomposites for

\section{Magneto-Scaffolds}

Hussein Awada ${ }^{\ddagger *}$, Assala Al Samad ${ }^{*}$, Danielle Laurencin ${ }^{\dagger *}$, Ryan Gilbert $^{\bar{T}}$, Xavier Dumail ${ }^{\dagger}$, Ayman El Jundi ${ }^{¥}$, Audrey Bethry ${ }^{¥}$, Rebecca Pomrenke ${ }^{\bar{\tau}}$, Christopher Johnson ${ }^{\bar{T}}$, Laurent

Lemaire $^{\S}$, Florence Franconi ${ }^{\S}$, Gautier Félix ${ }^{\dagger}$, Joulia Larionova ${ }^{\dagger}$, Yannick Guari ${ }^{\dagger}$, Benjamin Nottelet $^{¥ *}$

${ }^{\ddagger}$ IBMM, Université de Montpellier, CNRS, ENSCM, Montpellier, France.

†ICGM, Université de Montpellier, CNRS, ENSCM, Montpellier, France.

${ }^{\bar{\top}}$ Rensselaer Polytech Inst, Dept Biomed Engn, Ctr Biotechnol \& Interdisciplinary Studies, Troy, NY 12180 USA

§Micro \& Nanomédecines Translationnelles-MINT, UNIV Angers, INSERM U1066, CNRS

UMR 6021, Angers, France ; PRISM Plate-forme de recherche en imagerie et spectroscopie multi-modales, PRISM-Icat Angers, France 


\section{ABSTRACT}

Composites combining superparamagnetic iron oxide nanoparticles (SPIONs) and polymers are largely present in modern (bio)materials. However, while SPIONs embedded in polymer matrices are classically reported, the mechanical and degradation properties of the polymer scaffold are impacted by the SPIONs. Therefore, the controlled anchoring of SPIONs onto polymer surfaces is still a major challenge. Herein, we propose an efficient strategy for the direct and uniform anchoring of SPIONs on the surface of functionalized-polylactide (PLA) nanofibers via a simple free ligand exchange procedure to design PLA@SPIONs core@shell nanocomposites. The resulting PLA@SPIONs hybrid biomaterials are characterized by electron microscopy (SEM and TEM) and EDXS analysis, to probe the morphology and detect elements present at the organic/inorganic interface, respectively. A monolayer of SPIONs with a complete and homogeneous coverage is observed on the surface of PLA nanofibers. Magnetization experiments show that magnetic properties of the nanoparticles are well-preserved after their grafting on the PLA fibers and that the size of the nanoparticles does not change. The absence of cytotoxicity, combined with a high sensitivity of detection in MRI both in vitro and in vivo make these hybrid nanocomposites attractive for the development of magnetic biomaterials for biomedical applications.

KEYWORDS: core@shell nanocomposite, hybrid biomaterial, iron oxide nanoparticles, poly(lactide) nanofibers, MRI and magnetic properties. 


\section{INTRODUCTION}

Superparamagnetic iron-oxide nanoparticles (SPIONs) are utilized in a number of biomedical applications including diagnostics (with commercially available contrast agents like dextrancoated SPIONs Sienna+(®), drug delivery, ${ }^{1-2}$ magnetofection, ${ }^{3-4}$ therapeutic hyperthermia, ${ }^{5}$ cell tracking and cell trapping, ${ }^{6-10}$ proteins expression, ${ }^{11-12}$ tissue engineering ${ }^{13-14}$ and combinations thereof in the frame of theranostic approaches. ${ }^{15-17}$ This success is of course explained by the magnetic properties of SPIONs but also by their low, if any, cytotoxicity in comparison to other magnetic materials. ${ }^{18}$ In most biomedical applications, SPIONs are combined with polymers in order to enhance their efficacy. Polymer/SPION systems can be divided in three main categories (Scheme 1): polymer-coated SPIONs, SPION-loaded nanoparticles and SPION-loaded composites.

The first category, polymer-coated SPIONs, has been developed as a response to the instability of SPIONs in water (at neutral $\mathrm{pH}$ ) or in physiological fluids, as they rapidly aggregate and precipitate out of solutions. As a consequence, a steric or electrostatic stabilization provided by polymer coatings is required to ensure colloidal stability, and the functionalization of SPIONs with polymers has been the focus of a large number of research studies and reviews. ${ }^{19-21}$ Generally, biocompatible functional polymers are grafted onto the inorganic particle through an anchoring group, such as an amine, carboxylic acid or phosphonic acid. A large variety of polymers have been used for this purpose, including biopolymers like dextran, ${ }^{22-23}$ chitosan, ${ }^{24}$ hyaluronic acid, ${ }^{5}$ but also synthetic polymers like poly(ethylene glycol) (PEG), ${ }^{25-27}$ polyamidoamine, ${ }^{28}$ polyglycerol, ${ }^{29}$ polyoxazoline, ${ }^{30}$ poly(dimethylaminoethyl methacrylate) ${ }^{31}$ or polypeptides/polypeptoids. ${ }^{32-33}$ 
In the second category, SPION-loaded nanoparticles, polymers are used as polymeric cargos for SPIONs, as they allow high local concentrations of magnetic materials and enhanced relaxivities to be reached, as well as concomitant drug loading. ${ }^{34-35}$ Among these systems, magnetic micelles and magnetic nanospheres based on polylactide (PLA) or other classical aliphatic polyesters have attracted much attention due to the long track record of these polymers in the biomedical field. ${ }^{36}$ As for drug delivery systems, while initial studies used simple PLA or PEG-b-PLA diblock copolymers to encapsulate SPIONs, ${ }^{37-39}$ the trend is currently to move towards more complex macromolecular designs to reach higher functionalities and a better control over the delivery thanks to temperature- or redox-sensitive copolymers ${ }^{40-41}$ or to cell-specific targeting moieties for dual targeting strategies. ${ }^{42-43}$ To ensure a compartmentalized localization of SPIONs and hydrophilic drugs, magnetopolymersomes have also been developed, as illustrated by the recently reported methicillin-loaded mPEG-b-polylactide (mPEG-b-PLA) magnetopolymerosmes used for the treatment of medical device-associated infections. ${ }^{44}$

On a larger scale, the last family of polymer/SPION materials are composite materials in which the SPIONs are embedded in a macroscopic polymer matrix for sorption, imaging or (bio)sensors applications. ${ }^{45-46}$ In particular, magnetic scaffolds are becoming an important class of scaffolds as they find a number of applications in tissue engineering, allowing for improved bone and tissue repair, as well as drug delivery. Various types of SPION-loaded scaffolds are therefore found in the literature, ranging from drug releasing soft in situ-gelling magnetic gels, ${ }^{47}$ to fibers with magnetically tunable mechanical properties ${ }^{48}$ or microrods that allow remote manipulation for improved in vivo integration. ${ }^{13}$ In this context, one of the major classes of magnetic scaffolds are the nanoparticle-nano/microfiber composites. ${ }^{49}$ To prepare iron-oxide loaded composite fibers, three techniques based on electrospinning (ES) are classically reported: (i) the most common one 
relies on the introduction of pre-synthesized SPIONs in a polymer solution prior to ES, (ii) the second one involves the inclusion of a precursor in the fibers which undergoes a post-ES process to yield the SPIONs within the fibers, and (iii) the most recent one uses in situ synthesis techniques where SPIONs are synthesized during the ES process or in the solution to be electrospun. ${ }^{50}$ To the best of our knowledge, in the frame of biodegradable polymers, only the first technique (i. e. dispersion of SPIONs in polymer solution followed by ES) is used on a practical perspective: this has been exemplified recently in works involving PLA or PCL scaffolds for faster bone or tissue repair under external magnetic fields, which stimulate cell proliferation and secretion of new extracellular matrix. ${ }^{51-54}$ However, this synthetic approach is associated with changes in size, shape and mechanical properties of the composite fibers in comparison to their non-loaded counterparts, which leads to an unwanted increase in their hydrophilicity and degradation rate.

Coated-SPIONs SPION-loaded nanoparticles SPION-loaded composites

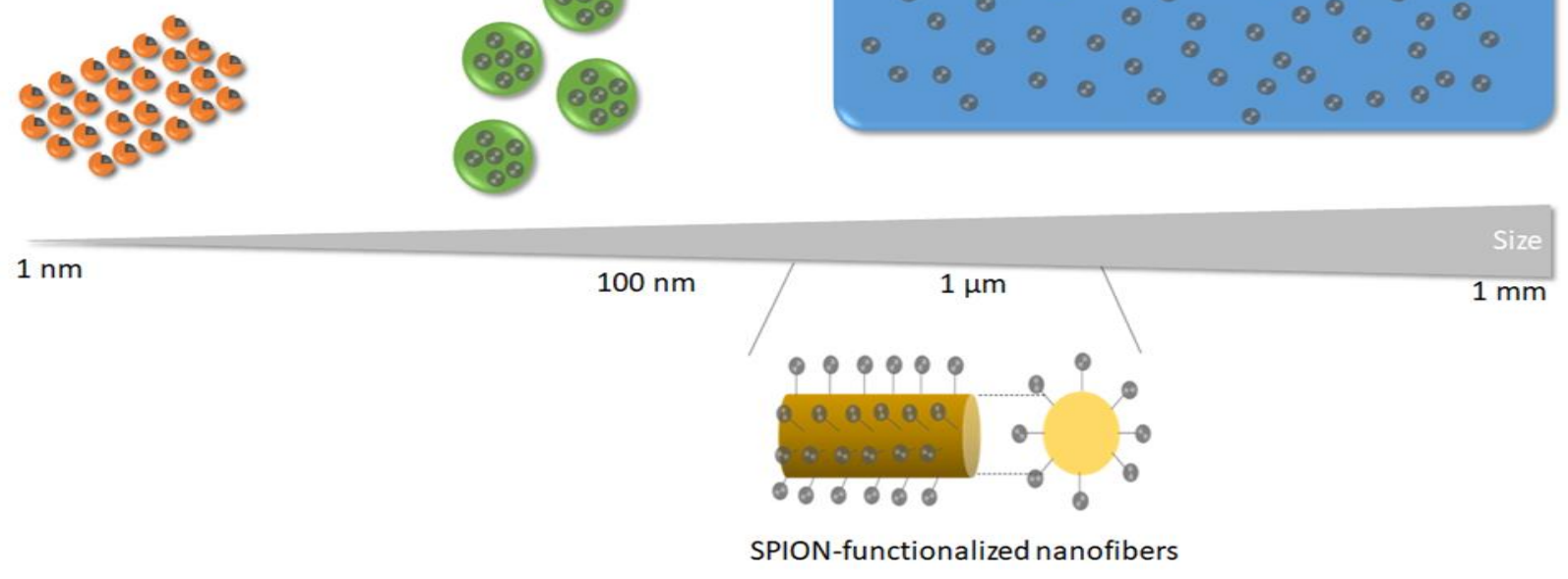

Scheme 1. Illustration of the different types of materials combining SPIONs and polymers reported in the literature (top), and of the SPION-functionalized nanofibers aimed herein (bottom). 
In order to minimize the changes in properties of the hybrid nanomaterials, while still taking advantage of the presence of magnetic nanoparticles, a last and less explored strategy consists in immobilizing the SPIONs at the surface of the polymeric fibers (Scheme 1, bottom). This has been achieved in the past by precipitation of iron oxide to the surface of the biomaterials.${ }^{55}$ However, two major drawbacks are associated with this approach: (i) the particles' morphology and aggregation during precipitation is poorly controlled and leads to large clusters rather than discrete particles, and (ii) the reaction conditions used for SPION formation are not compatible with a wide range of biopolymers. For these reasons, the covalent anchoring of pre-synthesized SPIONs onto the surface of biomaterials appears as a challenging and yet highly attractive alternative to yield SPION-functionalized nanomaterials. To the best of our knowledge, only two structures have been reported so far in this context, and yet none of them focused on polymer electrospun fibers and magnetic scaffolds, despite the interest such a combination would have for biomedical applications. Various groups prepared dual-mode contrast agents (ultrasound and MRI) by anchoring via reductive amination SPIONs exposing amine functions to the external surface of poly(vinyl alcohol)-shelled microbubbles made of aldehyde telechelic modified PVA. ${ }^{56-58}$ The same approach was followed by Kuo et al., who immobilized amino-functional SPIONs at the surface of poly[(acrylic acid)-b-(N-isopropylacrylamide)-b-(acrylic acid)] by amidation. ${ }^{59}$

The purpose of this work is to report a new strategy for the preparation of SPION-biopolymer fiber magnetic scaffolds, in which the intrinsic properties of both the biopolymer and SPIONs are maintained, and a uniform distribution of SPIONs along the fibers is achieved. More specifically, we describe the anchoring of SPIONs on the surface of PLA electrospun nanofibers by a combination of thiol-yne photoaddition and free ligand exchange procedure. The efficiencies of two synthetic strategies are first compared in terms of SPION- immobilization, SPION- 
aggregation and PLA surface integrity. The magnetic properties of the most promising PLA@SPIONs hybrid material are then evaluated, as well as their MRI visibility, and their biocompatibility, to guarantee their use in the frame of biomedical applications.

\section{RESULTS AND DISCCUSSIONS}

Preparation of hybrid PLA@SPIONs nanofibers. For the preparation of PLA@SPIONs hybrid biomaterials, the first step consisted in preparing a surface-modified PLA scaffold which exposes alkyne functionalities. Our previously published protocol was used for this purpose: the procedure involves a controlled anionic activation of the polymer in a $\mathrm{THF} / \mathrm{Et}_{2} \mathrm{O}$ solvent mixture using lithium diisopropyl amide (LDA) as strong base under inert atmosphere and allows the covalent immobilization of propargyl groups in $\alpha$-position of the carbonyl. ${ }^{60-61}$ In this work, the first objective was thus to test this strategy on PLA electrospun nanofibers, whose small size and high specific surface area were expected to render this modification procedure more delicate, by degrading the PLA fibers or by significantly modifying their morphology. The first experiments performed under standard conditions ${ }^{61}$ confirmed the fragility of the nanofibers, as the degradation of the PLA nanofibers was indeed observed (data not shown). Optimization of the conditions was therefore necessary, by reduction of LDA concentration (Table S1 entries 1 and 2 in the Supporting Information) and also of the THF content in the solvent mixtures (reduced from $25 \%$ to $5 \%$ volume ratio - see Table S1 entry 3). However, even under these milder conditions, the integrity of the fibers was not fully maintained after anionic activation, as some fibers were still cut even with the lowest THF content (5\% volume) (Figure 1B). In order to avoid any damage, the propargylation was finally carried out in pure $\mathrm{Et}_{2} \mathrm{O}$ (Table $\mathrm{S} 1$ entry 4). Under this condition, no degradation of nanofibers was observed by electron microscopy (Figures 1C). This was further confirmed at the 
macromolecular level with size exclusion chromatography (SEC) analyses (Figure S1 in the Supporting Information), showing very similar chromatograms before and after functionalization, with an average molecular weight of 78000 g.mol-1 . With a goal of assessing the presence and availability of the alkyne groups introduced at the surface of the PLA nanofibers, we further reacted propargylated PLA fibers with 9-azidomethyl anthracene (fluorescent molecule) in a water/acetone mixture under classical aqueous copper(I)-catalyzed alkyne-azide cycloaddition (CuAAC) conditions. ${ }^{60}$ Fluorescence microscopy images were recorded (Figures 1D, 1E, 1F), confirming the damage and disorganization of the fibers propargylated in the $\mathrm{Et}_{2} \mathrm{O} / \mathrm{THF}(95 / 5 \mathrm{~V} / \mathrm{V})$ mixture (Figure 1E), in contrast to those propargylated in pure $\mathrm{Et}_{2} \mathrm{O}$ which remain intact (Figure 1F). These images also show that the anthracene attachment onto the fibers was homogeneous, thereby proving that propargyl groups cover the whole fiber surface and that they are still reactive despite their immobilization on the PLA surface. Most importantly, no fluorescence was observed when reacting pristine PLA nanofibers (without alkyne groups) with the fluorescent molecule under the same CuAAC conditions (Figure 1D), thereby confirming that the fluorescence observed in Figures $1 \mathrm{E}$ and $1 \mathrm{~F}$ was only due to covalently bound anthracene molecules and not to adsorbed ones. 

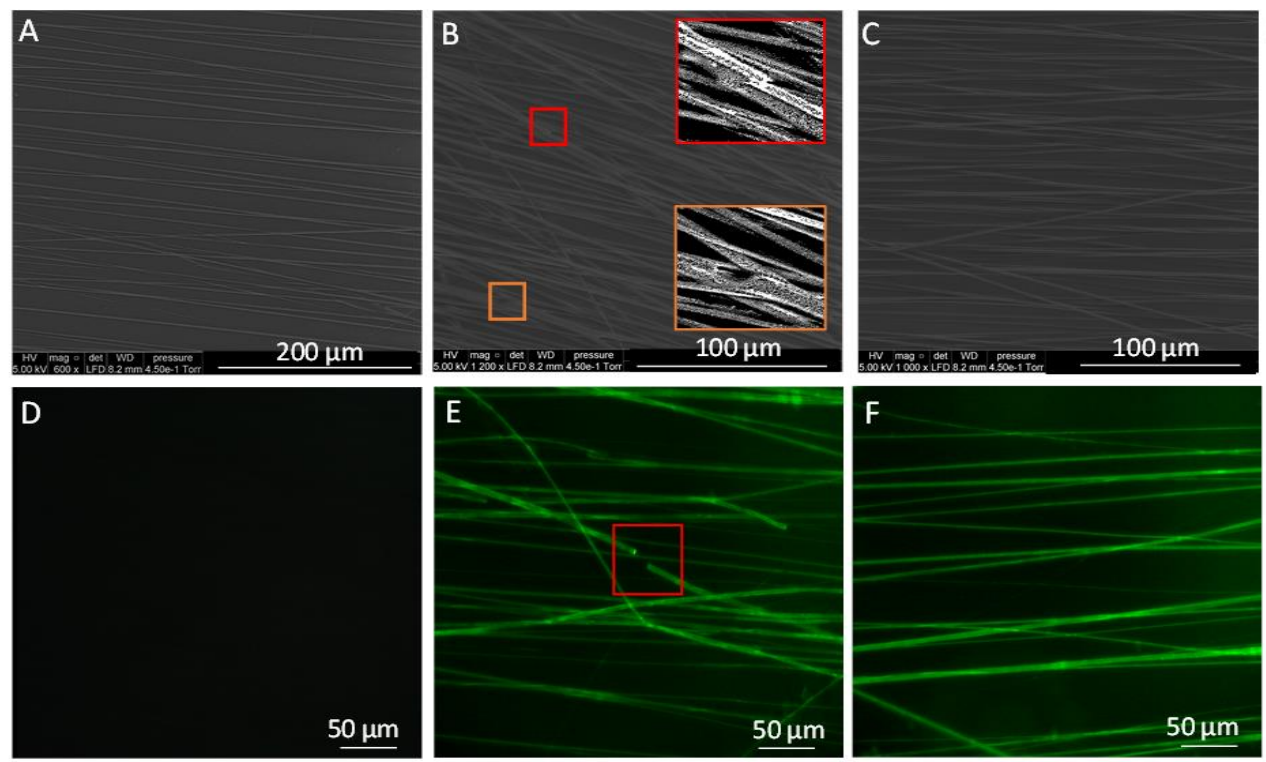

Figure 1. Impact of chemical modification of PLA nanofibers and presence of alkyne functionality were evaluated by scanning electron microscopy (A-C) and fluorescence microscopy (D-F), respectively. Pristine PLA nanofibers (A, D) are compared with PLA nanofibers modified in $\mathrm{Et}_{2} \mathrm{O} / \mathrm{THF}(95 / 5)$ mixture $(\mathrm{B}, \mathrm{E})$ and PLA nanofibers modified in pure $\mathrm{Et}_{2} \mathrm{O}(\mathrm{C}, \mathrm{F})$. Insets in $(\mathrm{B}$, E) correspond to magnifications of the highlighted areas where cuts and defaults on the nanofibers are visible.

Two synthetic strategies based on metal-free catalyst reactions were then tested to prepare the PLA@SPIONs hybrid magnetic scaffold (Scheme 2). In a first approach (strategy 1), thiolfunctionalized SPIONs were prepared via a free ligand exchange procedure (1st step) and then immobilized at the surface of alkyne-functionalized PLA electrospun nanofibers by thiol-yne photoaddition (2nd step). This was done by reacting oleic acid functionalized SPIONs with a bifunctional thiol-phosphonic ligand (12-mercaptododecylphosphonic acid): the stronger affinity of the phosphonic group for metal oxides allows the oleic acid shell to be removed from the surface of the SPIONs. ${ }^{62}$ For these nanoparticles $(\mathrm{d}=18 \mathrm{~nm}$ ) (Figure S2), a grafting density of thiol- 
phosphonic ligands of 2.5 molecules.nm ${ }^{-2}$ was calculated from TGA analysis (Figure S3). The coupling reaction between PLA nanofibers and SPIONs was subsequently performed under UVirradiation in a cyclohexane/acetone mixture $(3: 1),{ }^{61}$ in order to both keep the integrity of the PLA nanofibers and achieve an efficient thiol-yne photoaddition.

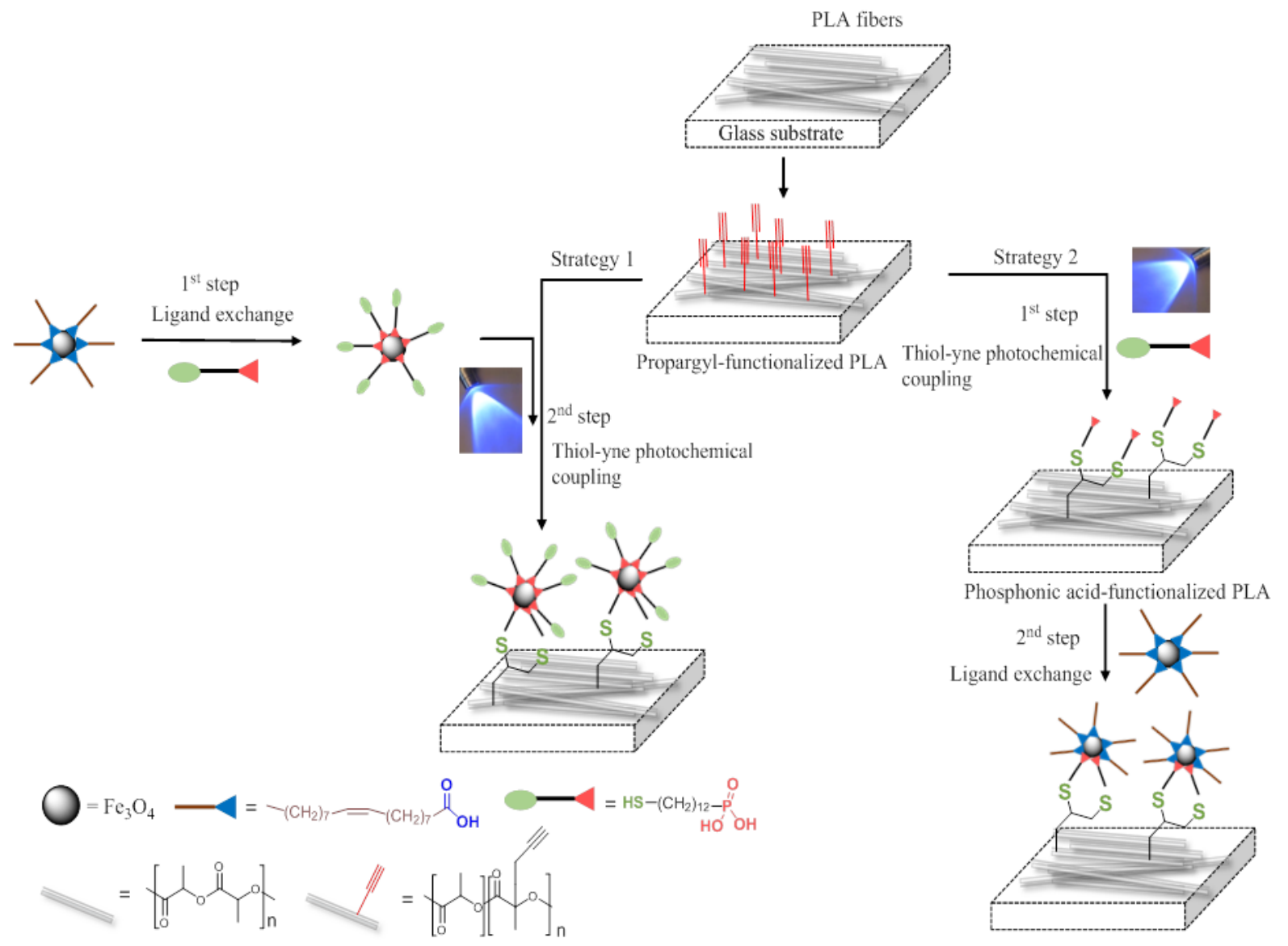

Scheme 2. Comparison of the two grafting strategies for the preparation of SPION-functionalized electrospun PLA nanofibers (PLA@SPIONs). For clarity of this representation, the nanoparticles and grafted molecules are not shown at scale here. 
In the second strategy, a reverse procedure was followed. The thiol-phosphonic ligand was first covalently bound to the alkyne-functionalized PLA electrospun nanofibers by photoaddition click chemistry $\left(1^{\text {st }}\right.$ step$)$, and then a ligand exchange procedure between oleic acid ligands initially present at the surface of the SPIONs and the phosphonic groups exposed at the periphery of the fibers ( $2^{\text {nd }}$ step). This was done by immersing the PLA fibers isolated after thiol-yne photoaddition in a solution of oleic-acid functionalized SPIONs in acetone/cyclohexane $(1 / 3, \mathrm{~V} / \mathrm{V})$ at room temperature for 24 hours.

Morphological and structural analysis of the hybrid materials. For the samples obtained by the two different strategies, SEM and EDXS analyses were first used to probe the surfacemorphology of the fibers and to evaluate the efficiency of SPION immobilization. For strategy 1, different synthetic conditions were tested (Table S2 and Figure S4). In all cases, despite the short irradiation times used ( 5-10 min), only the presence of aggregated particles was observed, and no iron was detected at the surface of the fibers in regions which were free of aggregates (Figure S5). A possible explanation is that the magnetic nature of the SPIONs combined to the high amount of thiol groups at their surface brings the nanoparticles in close proximity to each other, resulting in the formation of micron-sized clusters that are stabilized by the fast creation of disulfide bonds under UV irradiation. ${ }^{63}$ Normally, for monothiol functional molecules, the disulfide bond formation/cleavage should be a fast and reversible process under UV irradiation, but here, due to the magnetic properties of the nanoparticles and the large number of disulfide bonds formed between a SPION and its neighbors, it was not the case. Additional tests were thus performed by adding a reducing agent tris(2-carboxyethyl)phosphine (TCEP) in the reaction medium to cleave these disulfide bridges. However, a significant number of aggregates was still observed (Figure 
S4), which is a major drawback for biomedical applications, as it will significantly affect the properties of these hybrid nanofibers in vivo.

For the samples prepared according to strategy 2 (Scheme 2), EDXS analyses of the phosphonic acid-grafted intermediate revealed the presence of $\mathrm{P}$ and $\mathrm{S}$ elements with a ratio approximately equal to 1 at different scanning areas of the nanofiber surfaces (Figure 2B). This suggests the homogeneous grafting of the phosphonic ligand all over the nanofibers. Moreover, the morphology of the PLA nanofibers after modification is still preserved, as shown by SEM (Figure 2B). More importantly, for the subsequent SPION-grafting step, even for relatively long reaction times (24 h), the desired targeted scaffold was obtained without any presence of aggregates (Figure 2C). Indeed, the presence of $\mathrm{Fe}$ is detected by EDXS at different scanning areas (with similar ratios with respect to $\mathrm{P}$ and $\mathrm{S}$ when looking at different parts of the fibers), and high magnification SEM images of the nanofiber surfaces still reveal "smooth" surfaces (Figure S6), suggesting that the grafting of the SPIONs is homogeneous. As this stage, it is important to note that the cleaning procedure was carefully optimized to ensure that the Fe detected by EDXS did not correspond to residual physisorbed particles. This can be seen in the EDXS and SEM analyses of the control sample (Figure 2A), as no Fe was detected at the surface of the nanofibers. 


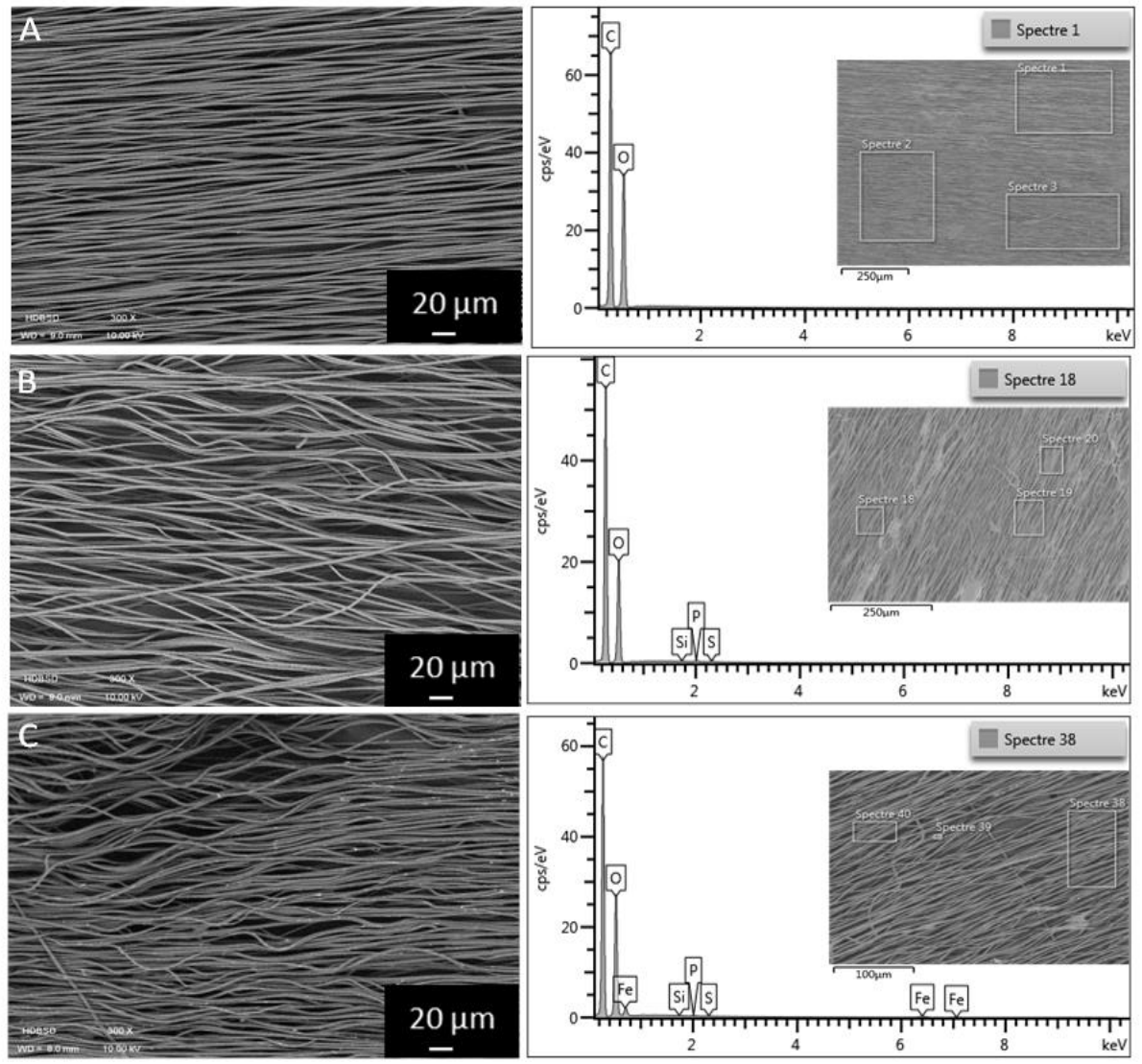

Figure 2. Visualization of SPIONs quasi-monolayer at the surface of PLA nanofibers. SEM and EDX analysis corresponding to strategy 2. A) Control sample, B) phosphonic acid functionalized PLA via thiol-yne photoaddition and C) PLA@SPIONs via ligand exchange procedure.

In order to further characterize the PLA@SPIONs hybrid nanofibers obtained using strategy 2, TEM analyses were carried out to determine their structure and morphology, notably at the organic-mineral interface. A homogeneous and complete coverage of SPIONs all over the fibrous scaffold was observed in TEM images (Figure 3). More specifically, on the longitudinal section, the full coverage of the PLA nanofiber surface was visible, with some nanoparticle clumps resulting from the sample preparation and the roughness of the PLA surface (Figure 3A). The cross section further confirmed the full and homogeneous coverage of PLA nanofibers by SPIONs which form a quasi-monolayer at the surface. To further study the stability of iron oxide shell, the 
PLA@SPIONs film was immersed in a phosphate-buffered saline solution for 3 days at $37{ }^{\circ} \mathrm{C}$. The atomic percentage of Fe element was constant according to EDXS analysis (Table S3). This confirms the strong interactions at the interface of the prepared hybrid core@shell material.
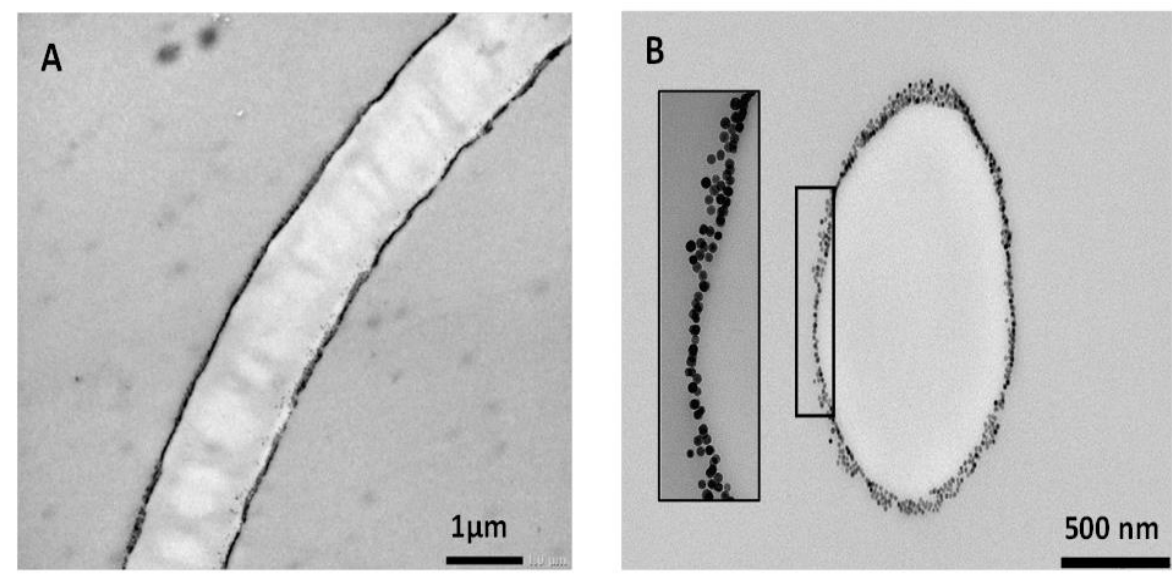

Figure 3. Visualization of SPIONs quasi-monolayer at the surface of PLA nanofibers. TEM images of PLA@SPIONs nanocomposites obtained with strategy 2 with A) longitudinal section and B) transverse section.

Thermal gravimetric analyses (TGA), were subsequently performed in order to examine the degradation of PLA@SPIONs hybrids and to quantify the amount of paramagnetic nanoparticles linked to the PLA surface (Figure S7). While the PLA polymer alone displayed a total thermal degradation between $280^{\circ} \mathrm{C}$ and $400^{\circ} \mathrm{C}$, the PLA@SPIONs composites were found to degrade between $240^{\circ} \mathrm{C}$ and $360^{\circ} \mathrm{C}$. The residual mass of $8 \%$ observed in the latter case attests of the overall amount of SPIONs anchored at the surface of the PLA nanofibers. It is to note that examples of SPIONs/polymer composite biomaterials that have been described for biomedical applications contain SPIONs in similar ratios, with typical values in the range 2.5 to $20 \mathrm{wt} \%$, with most biomaterials containing SPIONs at ca. $10 \mathrm{wt} \%{ }^{47-49,51,53-54,59}$ 


\section{Magnetic properties of the PLA@SPIONs composites obtained by strategy 2. The}

PLA@SPIONs composites shown in Figure 3 not only possess a well-defined interface, but also significantly react to the presence of magnetic fields. As a first evidence of this, the mobility of the nanofibers and their attraction by a magnet was analyzed (see Figure S8, Movies S1 and S2). To go one step further, the magnetic properties of the hybrid composite were investigated by using a SQUID-MPMS magnetometer. The PLA@SPIONs fibers were orientated parallel and perpendicular to the applied magnetic field directions. In order to demonstrate the impact of SPIONs' alignment on the PLA fibers, the magnetic properties of the composites were compared with the ones of the non-grafted nanoparticles. First of all, the temperature dependence of the magnetization in Zero Field Cooled (ZFC)/Field Cooled (FC) modes for PLA@SPIONs was measured in the temperature range $1.8-300 \mathrm{~K}$ (Figure 4A). As expected, the ZFC curve increases as the temperature decreases, reaches the maximum at $214 \mathrm{~K}\left(\mathrm{~T}_{\max }\right)$ and then decreases, while the FC curve separates from the $\mathrm{ZFC}$, decreases below $\mathrm{T}_{\max }$ and reaches the plateau below $100 \mathrm{~K}$. The $\mathrm{ZFC} / \mathrm{FC}$ curves of the pristine SPIONs (Figure S9) show very similar shape with $\mathrm{T}_{\max }=207 \mathrm{~K}$, which indicates that the magnetic properties of the nanoparticles are well-preserved after their grafting on the PLA fibers and that the size of the nanoparticles did not change. Secondly, the magnetization $v s$ applied magnetic field was performed at $300 \mathrm{~K}$ for samples where magnetic fibers were oriented parallel and perpendicular to the field directions. The curves do not present a hysteresis, indicating that the nanoparticles are not blocked at room temperature, which is in agreement with the behavior of non-grafted nanoparticles (Figure S9). In addition, the values of the saturation magnetization are in agreement with the amount of SPIONs found by the TGA measurements. 

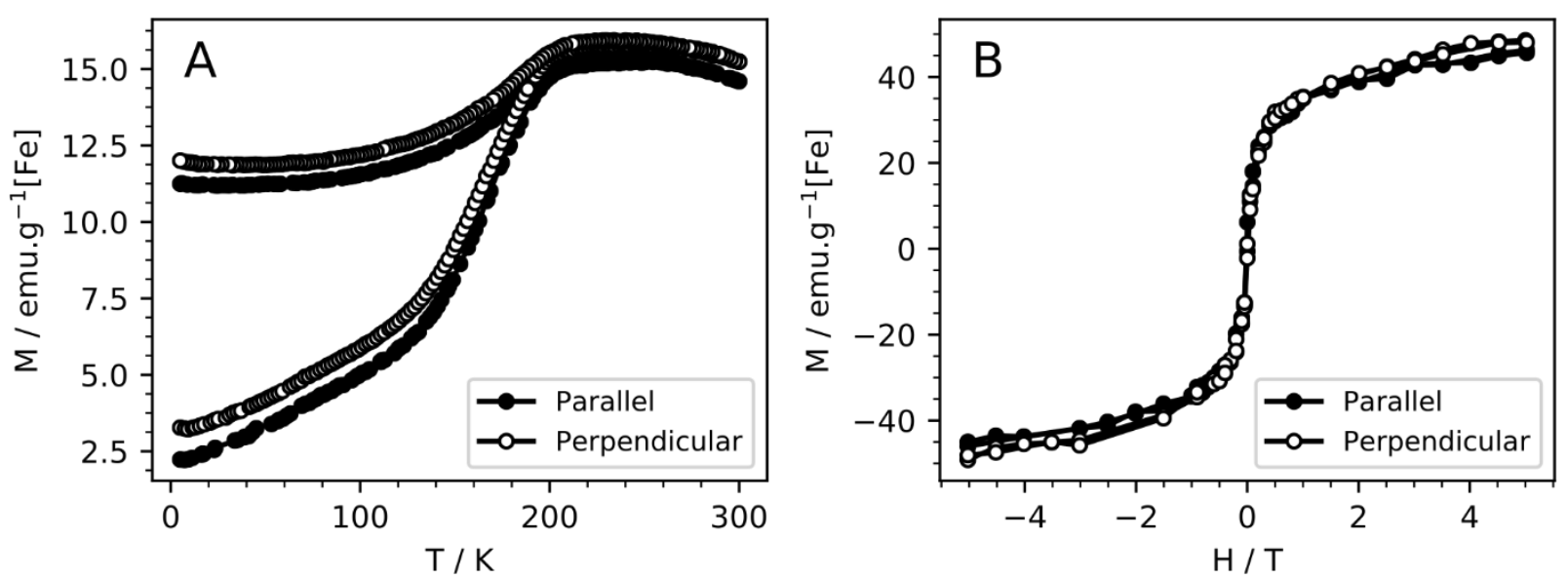

Figure 4. Magnetic properties of PLA@SPIONs. A) FC/ZFC curves performed with an applied magnetic field of $0.1 \mathrm{~T}$ for PLA@SPIONs with fibers parallel and perpendicular to the direction of the field; B) Field dependence of the magnetization for PLA@SPIONs measured at 300 K with fibers parallel and perpendicular to the direction of the magnetic field. The values of the magnetization are given as emu per $\mathrm{g}$ of $\mathrm{Fe}$.

MRI visibility of hybrid nanofibers prepared according to strategy 2. Due to their high magnetic moments, SPIONs can be used as superparamagnetic contrast agents and therefore be observed as hyposignals on T2, T2*-weighted images, and as phase disturbances on susceptibility weighted imaging (SWI) phase-filtered images. The relaxivity measurements for the non-grafted SPIONs have previously been reported, yielding a transverse relaxivity value of $\mathrm{r}_{2}=289 \mathrm{mM}^{-1} \mathrm{~s}^{-1}$ and a $r_{2} / r_{1}$ ratio of $27,{ }^{64}$ which makes them promising as potential $T_{2}$-weighted MRI contrast agents. As observed on Figure 5A, pristine PLA nanofibers without SPIONs could barely be seen on the T2 weighted image. The thin line observed corresponds in fact to a lack of signal within the embedding gel used for the analysis, due to the presence of fibers with ultrashort T2. However, when PLA@SPIONs nanofibers (Figure S10) were analyzed (Figure 5D), a clear hyposignal around the fibers was present. The difference between PLA@SPIONs and pristine nanofibers is 
even more pronounced on the T2*-weighted (Figures 5B, E) and SWI phase filtered (Figures 5C, F) images, both methods being intrinsically more sensitive to SPION susceptibility effects. Indeed, the PLA@SPIONs nanofibers magnetic susceptibility disturbs the magnetic field homogeneity to a much larger extend than their real size, inducing the large susceptibility effects visible on the images. This set of results clearly demonstrates that PLA@SPIONs nanofibers can be imaged using the clinically relevant T2 MRI sequences.

To go one step further, pre-clinical experiments on rats implanted with PLA@SPIONs nanofibers were performed using a standard T2-weighted spin echo sequence and a pre-clinical MR scanner. For this purpose, PLA fibers and PLA@SPIONs fibers were implanted in the peritoneal cavity of rats. One week later, in vivo MR imaging on the anaesthetized animal were performed, which showed a strong and fine hypointense line on the implantation site of the PLA@SPIONs fibers (Figure 5G). In contrast, the nanofibers without SPIONs that were implanted on the other side of the rat abdomen could barely be seen, and required the analysis of the images by trained radiologists to be depicted. This first result confirms the potential of the PLA@SPIONs as biomaterials for in vivo implantation. Long-term in vivo studies dedicated to MRI, inflammatory response and interaction between tissue and SPIONs will be carried out in the near future on these systems. 


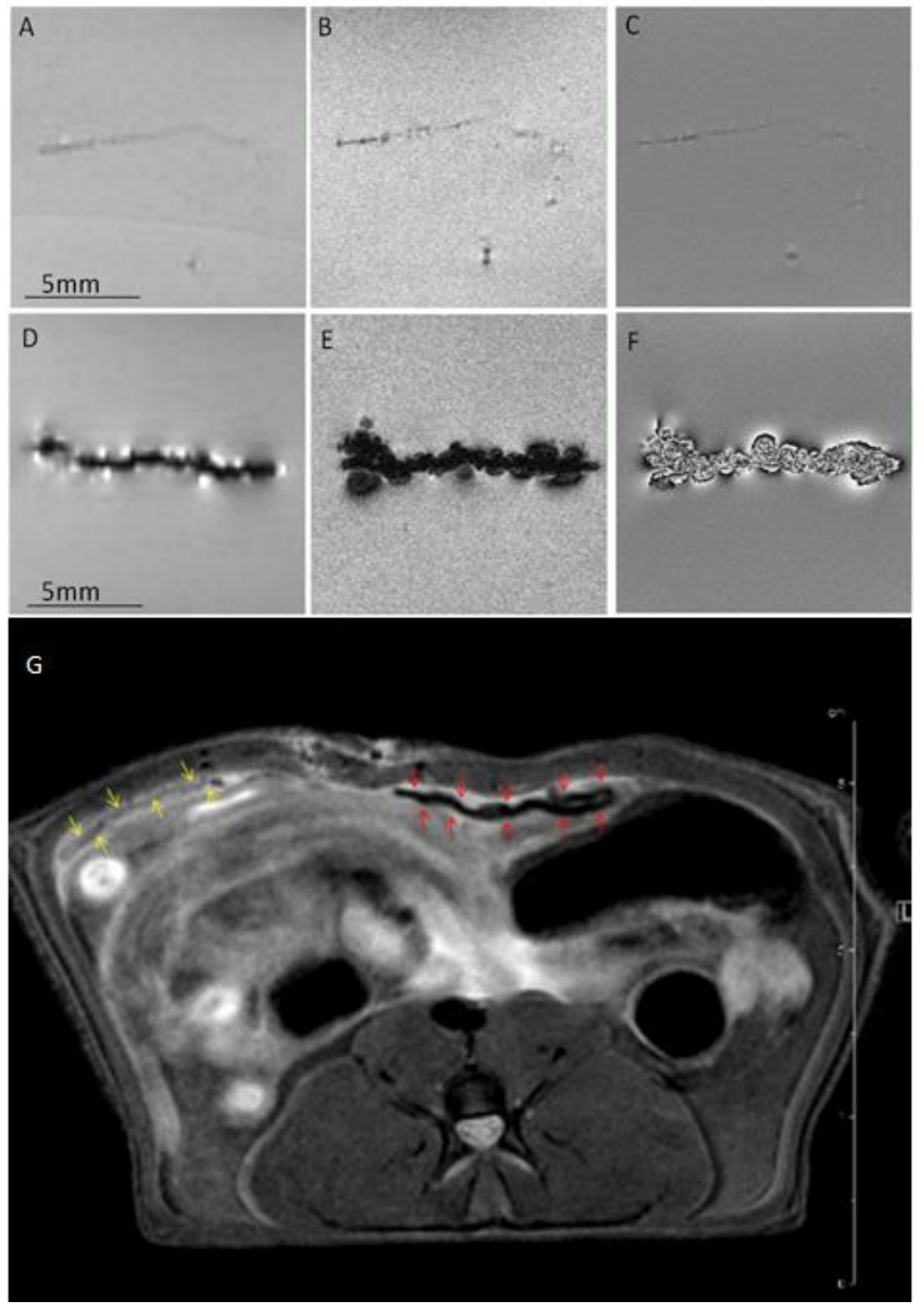

Figure 5. Magnetic resonance imaging of PLA@SPIONs. In vitro MRI analyses of PLA nanofibers without (A-C) or with (D-F) SPION functionalization: T2 weighted (A, D), T2* weighted $(\mathrm{B}, \mathrm{E})$ and SWI filtered phase $(\mathrm{C}, \mathrm{F})$ images are displayed. In vivo T2-weighted $\mathrm{MR}$ images acquired 1 week after implantation of nanofibers within the abdominal cavity of rats $(\mathrm{G})$. Red arrows point to the nanofibers grafted with SPIONs and the yellow arrows to the nanofibers without any contrast agent. 
Cytocompatibility of hybrid nanofibers prepared according to strategy 2. Given the potential applications of the PLA@SPIONs nanofibers as scaffolds, we were interested in evaluating the cytocompatibility of these hybrid biomaterials, which in addition to their MRIvisibility and magnetic susceptibility should also be nontoxic for cells. Cytocompatibility tests of the modified biomaterials were conducted using the L929 fibroblasts cell line. For proliferation studies, the evaluation was carried out on PLA@SPIONs films (Figure 6A). Indeed, when PLA nanofibers were used for this test, a bias of proliferation was induced by cells proliferating not only on the fibers but also between the fibers directly on the fibers support as shown in Figure S11.

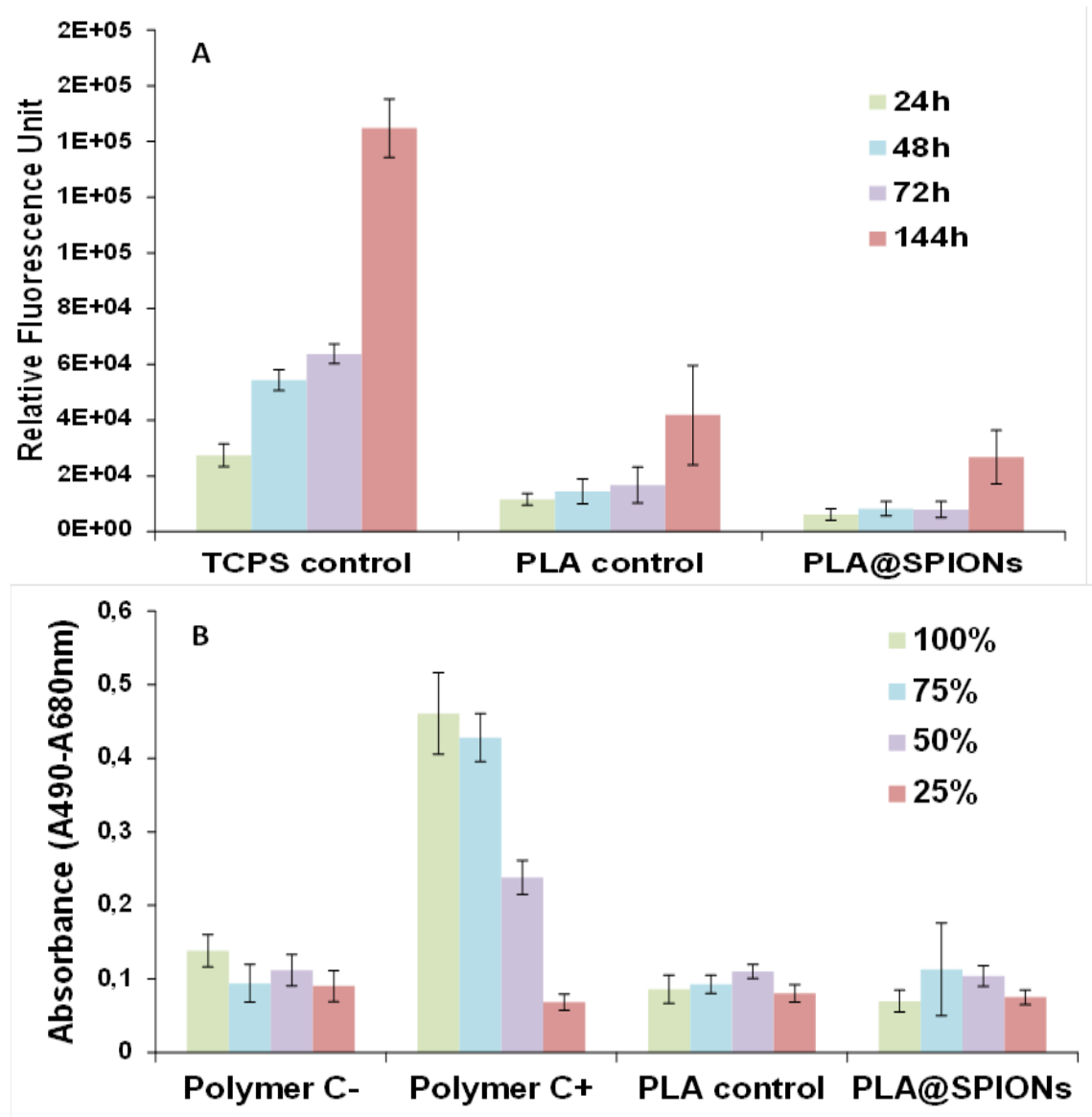

Figure 6. Evaluation of PLA@SPIONs cytocompatibility. (A) L929 proliferation on PLA@SPIONs surface compared to pristine PLA surface and TCPS positive control at 1,2,3, and 
6 days (data are expressed as means \pm SD and correspond to measurements with $n=5$ ); and (B) Cytotoxicity effects of polymer extracts on L929 cells after $24 \mathrm{~h}$ evaluated by Lactate Dehydrogenase (LDH) assay. PLA@SPIONs nanofibers are compared to pristine PLA nanofibers and to the negative control Polymer C- (RM-C High Density Polyethylene) and the positive control polymer C+(RM-B $0.25 \%$ ZDBC polyurethane). $100 \%$ corresponds to pure extract used as culture medium whereas $75 \%, 50 \%$, and $25 \%$ correspond to dilution of the pure extract with fresh culture medium to reach the defined concentration of extract. (Data are expressed as means $\pm \mathrm{SD}$ and correspond to measurements with $\mathrm{n}=9$ ).

Compared to TCPS positive control, proliferation was clearly limited on the PLA-based materials with 70 to $80 \%$ lower cell proliferation after 6 days. However, the proliferation of L929 cells was similar when cells were seeded onto PLA control and on the hybrid PLA@SPIONs, with no significant difference. Considering the fact that PLA is well known for its biocompatibility, this set of results clearly demonstrates that the PLA surface functionalized with SPIONs can be considered for cell contacting applications. To further confirm this with the PLA@SPIONs nanofibers, the cytotoxicity of the polymers was assessed according to the ISO 10993-12 international standard extract method with the extracts cytotoxicity being assessed by Lactate Dehydrogenase (LDH) assay. PLA@SPIONs nanofibers were compared to pristine PLA nanofibers and to the negative control Polymer C- (non cytotoxic RM-C High Density Polyethylene) and the positive control polymer C+ (cytotoxic RM-B 0.25\% ZDBC polyurethane) (Figure 6B). Only the extracts from polymer $\mathrm{C}+$ induced cytotoxicity, with liberation of LDH in growth medium which indicates cell membrane damages. On the opposite, for all concentrations of extract only low levels of LDH were detected for the pristine PLA nanofibers and the 
PLA@SPIONs nanofibers, without any significant difference compared to the negative control polymer C-. These results confirm that the immobilization of SPIONs on the PLA fibers following strategy 2 does not induce any cytotoxic effect for L929 cells, making them highly suitable for biomedical applications.

\section{CONCLUSION}

Overall, we have developed a synthetic methodology for the direct anchoring of iron oxide nanoparticles at the surface of PLA nanofibers. For this purpose, a bifunctional thiol-phosphonic ligand was used. The thiol group enabled a covalent attachment to the functionalized PLA surface by thiol-yne click chemistry under UV irradiation, while the phosphonic group allowed the SPIONs to be immobilized via ligand exchange. The morphology and structure of the hybrid PLA@SPIONs nanofibers were fully characterized by electron microscopy (SEM and TEM) and EDXS, revealing the homogeneous coverage of the nanofiber surface by a layer of nanoparticles. Cytotoxicity assays were performed, demonstrating the suitability of the PLA@SPIONs nanofibers for biomedical applications. Magnetic properties investigations confirmed that the size of the nanoparticles as well as their nature have not been altered during the grafting. On a more general perspective, compared to previous studies aiming at associating electrospun polymer fibers to magnetic nanoparticles for biomedical applications, we demonstrate here for the first time an efficient surface grafting method that not only maintains the initial fiber morphology (diameter \& length), but also avoids unwanted aggregation of magnetic nanoparticles. This synthetic approach is attractive as it can be readily adapted to other polyester scaffolds of different morphology and/or composition (eg. like PCL or PLGA, which can undergo the initial thiol-yne functionalization step), but more generally to any polymer substrate susceptible of being post-modified by a 
phosphonic group. Moreover, inorganic nanoparticles other than SPIONs can also be attached through the phosphonic group, thereby opening the way to an even broader range of biomaterials applications. Considering the potential of the PLA@SPIONs as biomaterials for in vivo implantation, long-term in vivo studies dedicated to MRI, inflammatory response and interaction between tissue and SPIONs will be carried out in the near future on these systems.

\section{METHODS}

Materials. Propargyl bromide ( $80 \mathrm{wt} \%$ in toluene), lithium diisopropylamide ( $2 \mathrm{M}$ in THF/heptane/ethylbenzene), 2,2-dimethoxy-2-phenylacetophenone (DMPA, >99\%), tris(2carboxyethyl)phosphine hydrochloride solution (TCEP, 0.5M, pH7), iron(III) oxide hydrated (catalyst grade, 30-50 mesh), oleic acid (90\% purity) and docosan (99\% purity) were purchased from Sigma-Aldrich. Oleylamine, with an approximate C18 content of 80-90\% (97\%), was purchased from Acros Organics. 12-mercaptododecylphosphonic acid was purchased from Sikemia. Poly-L-lactic acid (PLA100 noted PLA in the manuscript, Mw B78 kDa, NatureWorks, grade 6201D) was purchased from Cargill Dow LLC (Minnetonka, MN). Azido-functional anthracene was synthesized according to a previously described protocol. ${ }^{65}$ Absolute ethanol, cyclohexane, methanol, chloroform and acetone (99\% purity) were used as received. Dry THF was obtained by the solvent purification system PureSolve MD5 from Innovative Technology. Fresh diethyl ether $\left(\mathrm{Et}_{2} \mathrm{O}\right)$ was dried over molecular sieves prior to use.

Characterizations. Size exclusion chromatography (SEC) was performed at $30{ }^{\circ} \mathrm{C}$ with $\mathrm{THF}$ eluent at a flow rate of $1 \mathrm{~mL} \cdot \mathrm{min}^{-1}$ on Viscoteck GPC-Max VE 2001 equipment fitted with a $2 \times$ $30 \mathrm{~cm}$ long $5 \mathrm{~mm}$ mixed-C PLgel columns and coupled with a VE 3580 RI detector and a VE 3210 
UV/Vis detector. Typically, the polymer $(2 \mathrm{mg})$ was dissolved in THF $(0.5 \mathrm{~mL})$ and the resulting solution was filtered through a $0.45 \mathrm{~mm}$ Millipore filter before injecting $100 \mu \mathrm{L}$ of filtered solution. Calibration was against polystyrene standards. Scanning electron microscopy (SEM) studies were carried out on a Zeiss Evo HD15 scanning electron microscope equipped with an Oxford Instruments X-Max ${ }^{\mathrm{N}} \mathrm{SDD} 50 \mathrm{~mm}^{2}$ EDX detector. Before analysis, the samples were metallized with carbon. The observation of fibers after fluorescent labeling by anthracene was carried out on a Nikon 150231 fluorescence microscope. Transmission electron micrographs were recorded on a JEOL 1200EX transmission electron microscope, using an accelerating voltage of $100 \mathrm{kV}$. Ultrathin sections of the SPION-functionalized PLA fibers (30-100 $\mathrm{nm}$ in thickness) were obtained using a Leica ultracut ultramicrotome, after setting the sample in an LR-White resin. The thin sections were then placed on carbon-formvar coated copper grids. Thermal gravimetric analysis (TGA) was performed on a TGA Q50, TA Instruments at a heating rate of $10{ }^{\circ} \mathrm{C} \cdot \mathrm{min}^{-1}$ under air. Magnetic measurements were performed by using a Quantum Design MPMS-XL SQUID magnetometer working in the $1.8-350 \mathrm{~K}$ temperature range with the applied magnetic field up to 7 Tesla. The measurements were done on powder samples for the non-grafted SPIONs and for four stacked PLA@SPIONs films oriented along the fibres direction and positioned parallel and perpendicular to the direction of the magnetic field. The data was also corrected for the sample holder and for the diamagnetic contribution of non-grafted PLA. The decrease in the magnetization value observed on the ZFC curve for the magnetic scaffolds as the temperature decreases (Figure 4) could be explained by the presence of residual non oxidized FeO. ${ }^{66}$

Electrospun PLA fiber fabrication and PLA film preparation. Electrospun fibers were prepared from an electrospinning solution of $8 \% \mathrm{wt} . / \mathrm{wt}$. high molecular weight poly(lactic acid) (PLA100; Ingeo biopolymer 6201D, NatureWorks, Minnetonka, MN, USA) dissolved in 
chloroform (288306, Sigma-Aldrich, St. Louis, MO) as previously described ${ }^{67}$ The parameters used during the electrospinning of aligned fibers were: $2.0 \mathrm{~mL} \cdot \mathrm{h}^{-1}$ pump rate, $1000 \mathrm{rpm}$ rotation speed, 20 min collection time, $15 \mathrm{kV}$ voltage drop, $32-33 \%$ humidity, $20-23^{\circ} \mathrm{C}$ temperature, and a $5 \mathrm{~mm}$ gap distance between the needle and the grounded collector. Fibers were electrospun directly onto glass coverslips (15 x 15 mm; Knittel Glass, Brausenweig, Germany). After electrospinning, the edges of the coverslips were dipped into a $4 \%$ wt. PLA/wt. chloroform solution to secure the aligned fibers to the coverslip. For in vivo implantation, the parameters used during the electrospinning of a mesh with a high grafting density of fibers were: $2.0 \mathrm{~mL} \cdot \mathrm{h}^{-1}$ pump rate, 60 min collection time, $12 \mathrm{kV}$ voltage drop, $20-23^{\circ} \mathrm{C}$ temperature, and a $15 \mathrm{~mm}$ gap distance between the needle and the grounded collector. The PLA films for biological assays were prepared as reported in the literature. ${ }^{68}$ In brief, $480 \mathrm{mg}$ of precursor were dissolved under magnetic stirring in a $\mathrm{CH}_{2} \mathrm{Cl}_{2} / \mathrm{CHCl}_{3}$ mixture (composed of $6 \mathrm{~g}$ of $\mathrm{CH}_{2} \mathrm{Cl}_{2}$ and $6 \mathrm{~g}$ of $\mathrm{CHCl}_{3}$ ). The mixture was stirred for 4 hours and then cast in a PTFE flat form, evaporating dish (VWR). The dish was then covered by perforated tin foil, allowing a slow evaporation of the solvent at room temperature overnight. This procedure resulted in a homogeneous plastic film of $1 \mathrm{~mm}$ thickness. After removal from the dish, the film was cut into disks of $12 \mathrm{~mm}$ of diameter.

Propargylation of PLA fibers deposited on glass substrates. In a flamed-dried 3-Neck Lab reactor with Lid and steel clamp, $100 \mathrm{~mL}$ of dry $\mathrm{Et}_{2} \mathrm{O}$ was introduced under argon atmosphere and the medium was cooled to $-20^{\circ} \mathrm{C}$. Then, a Teflon rack holding 10 glass plates covered by PLA 100 fibers was immerged into the solvent. The solution was saturated with argon for $15 \mathrm{~min}$, before LDA (2M solution, $1 \mathrm{~mL}, 2 \mathrm{mmol}$ ) was added. After $15 \mathrm{~min}$, a propargyl bromide solution (80 $\mathrm{wt} \%$ in toluene, $1 \mathrm{~mL}, 9 \mathrm{mmol}$ ) saturated with argon was injected, and the mixture was stirred for 
an additional $15 \mathrm{~min}$ at $-50^{\circ} \mathrm{C}$. The reaction was quenched with water and the rack was washed several times in a cold diethyl ether bath for $30 \mathrm{~min}$, before drying under argon atmosphere.

Preparation of Anthracene-functionalized PLA fibers through click chemistry. A glass plate bearing propargyl-functionalized PLA fibers was placed into mixture of distilled water/acetone solution (9/1), before addition of $100 \mathrm{mg}$ 9-azidomethyl anthracene (solubilized in $200 \mu \mathrm{L}$ of acetone), $300 \mu \mathrm{L}$ of an aqueous solution of copper sulfate (1M) and $600 \mu \mathrm{L}$ of an aqueous solution of sodium ascorbate $(1 \mathrm{M})$. The mixture was then gently stirred at room temperature during $24 \mathrm{~h}$ on an orbital shaker. Finally, the plate was washed by water and ethanol and then immersed several times in a bath of cyclohexane/acetone (3:1) ratio for $30 \mathrm{~min}$, and then dried under argon. The immobilization of anthracene and impact of the modification on the shape of PLA fibers was assessed by fluorescence microscopy.

Preparation of super magnetic iron oxide nanoparticles (SPIONs). Oleic acid-functionalized SPIONs were synthesized by method similar to those reported in the literature. ${ }^{69}$ Briefly, $0.18 \mathrm{~g}$ of FeOOH fine powder, $3.2 \mathrm{~g}$ of oleic acid and $5.00 \mathrm{~g}$ of $\mathrm{n}$-docosane were combined and evacuated for $30 \mathrm{~min}$. Then, the mixture was heated under argon at $340{ }^{\circ} \mathrm{C}$ for $1.5 \mathrm{~h}$. The reaction mixture was allowed to cool to approximately $50{ }^{\circ} \mathrm{C}$ and diluted with $15 \mathrm{~mL}$ of pentane. Furthermore, the nanoparticles were purified and isolated after successive dispersion-centrifugation cycles using diethyl ether: ethanol mixtures (2:1 and 1:1) ratio at 20,000 rpm for $10 \mathrm{~min}$, with removal of the supernatant containing organic components. The purification was repeated several times until the supernatant solution became colorless. Finally, the SPIONs were redispersed in cyclohexane (20 $\mathrm{mL})$ in the presence of additional oleylamine $(200 \mu \mathrm{L})$ and freshly used in further experiments. TEM analyses were performed on these nanoparticles, showing that their average diameter was $\sim 18 \mathrm{~nm}$. 


\section{Preparation of PLA@SPIONs hybrids: strategy 1}

\section{Step 1: Thiol-functionalized SPIONs (SPIONs-SH) through free ligand exchange}

procedure. Oleic acid-functionalized SPIONs were isolated by precipitation with ethanol and centrifugation cycles. Then, $\sim 0.55 \mathrm{mmol}$ of the functionalized SPIONs and $\sim 1.4 \mathrm{mmol}$ of HS- $\left(\mathrm{CH}_{2}\right)_{12}-\mathrm{P}(\mathrm{O})(\mathrm{OH})_{2}$ were heated to reflux in dry THF $(20 \mathrm{~mL})$ under Argon atmosphere, and the medium was stirred for $48 \mathrm{~h}$. After cooling to room temperature, the thiol-functionalized SPIONs were recovered after successive dispersion-centrifugation cycles with pentane and methanol. The desired product was then dried for 15 min under vacuum, and redispersed in chloroform.

Step 2: SPION-functionalized PLA fibers through thiol-yne photoaddition. In a flame-dried 3-neck Lab reactor with lid and steel clamp protected from light with aluminum foil, $(0.34 \mathrm{mg}$, $1.34 \mu \mathrm{mol}) \mathrm{DPMA}, 2 \mathrm{mg}$ of functionalized SPIONs and $80 \mathrm{~mL}$ of a cyclohexane/ acetone mixture (3:1 ratio) were added, prior to immersion of Teflon rack holding 10 glass plates covered by propargyl-functionalized PLA fibers. The reaction medium was saturated with argon, and then UV irradiation $\left(100 \mathrm{Mw} \cdot \mathrm{m}^{-2}\right)$ was carried out between 5 and $10 \mathrm{~min}$ after removal of the aluminum foil. The SPION-functionalized PLA fibers were cleaned by successive washings in a cyclohexane/acetone mixture and dried under argon. A change in color from white to light brown was clearly observed for the PLA fibers. The desired material was stored in the fridge before performing further characterizations. In an alternative procedure, the reductive agent TCEP was added to the suspension of SPIONs one hour before the UV activation to ensure the reduction of disulfide bonds potentially formed between the functionalized SPIONs-SH, and to help reduce disulfide bonds that may subsequently form upon UV irradiation. All other steps were kept identical. 


\section{Preparation of PLA@SPIONs hybrids: strategy 2}

\section{Step 1: Phosphonic acid-functionalized PLA fibers through thiol-yne photo addition. In a}

flame-dried 3-neck Lab reactor with lid and steel clamp protected from light and placed under argon atmosphere, 12-mercaptododecylphosphonic acid $(0.38 \mathrm{mg}, 1.34 \mu \mathrm{mol})$ and DMPA (0.34 $\mathrm{mg}, 1.34 \mu \mathrm{mol})$ were added to $80 \mathrm{~mL}$ of a cyclohexane/ acetone mixture (3:1 ratio). The solution was saturated with argon prior to immersion of a Teflon rack holding 10 glass plates covered by propargyl functionalized PLA fibers. Then UV irradiation $\left(100 \mathrm{~mW} . \mathrm{cm}^{-2}\right)$ was carried out for 10 min under gentle stirring. Following the thiol-yne photo-addition, PLA fibers were washed several times and dried under argon atmosphere.

Step 2: SPION-functionalized PLA fibers through free ligands exchange procedure. A 100 $\mathrm{mL}$ cyclohexane/ acetone mixture (3:1 ratio) was introduced to a flame-dried 3-neck Lab reactor with lid and steel clamp under argon atmosphere. Then, a Teflon rack holding 10 phosphonic acidfunctionalized PLA fibers was immersed in the solution before addition of an excess of freshly prepared oleic acid-functionalized SPIONs $(2 \mathrm{mg})$ dispersed in cyclohexane solution with a concentration of $1 \mathrm{mg} \cdot \mathrm{mL}^{-1}$ and sonicated in an ultrasonic bath for $30 \mathrm{~min}$. The reaction was protected from light and stirred for $24 \mathrm{~h}$ at room temperature under argon. The SPIONfunctionalized PLA fibers were cleaned by successive washings in a cyclohexane/acetone mixture, and dried under argon. A change in the color from white to light brown was clearly observable for the PLA fibers. The desired material was stored in the fridge before performing further characterizations.

Implantation of the grafted fibers and MRI imaging. Animal care and use were in accordance with the regulations of the French Ministry of Agriculture and approved by the Pays de la Loire Ethics in Animal Experimentation Committee under project number 01858.03. Animals were 
housed in a controlled and pathogen-free environment, with free access to food and water, at the University animal facility (SCAHU-Angers, France). The implantation of the fibers (functionalized or not) within the peritoneal cavity was performed on isoflurane anesthetized Sprague Dawley rats $(220-250 \mathrm{~g}, \mathrm{n}=4)$ (continuous flow of air $-0.8 \mathrm{~L} / \mathrm{min}-2 \%$ isoflurane (Piramal Healthcare, UK Limited, Northumberland, UK), according to a modified Rodeheaver procedure without caecal abrasion. ${ }^{70}$ Animals received a $30 \mu \mathrm{g} / \mathrm{kg}$ subcutaneous injection of Vetergesic ${ }^{\circledR}$ (buprenorphin, Sogeval, France) prior to surgery. As no signs of stress were observed, no additional injection of buprenorphine was performed. One week later, in vivo MR imaging on anaesthetized animal (isoflurane) were performed on a Bruker Biospec 70/20 system operating at a magnetic field of 7T (Bruker, Wissembourg, France) using a 72-mm diameter birdcage resonator and a respiratory triggered RARE sequence (effective repetition time TR $\sim 2000 \mathrm{~ms}$; rat breathing rate $30-35 / \mathrm{min}$; effective echo time $\mathrm{TE}_{\text {eff }}=23 \mathrm{~ms}$; $R A R E$ factor $=8 ; \mathrm{FOV}=55 \mathrm{~mm} \times 55 \mathrm{~mm}$; matrix $256 \times 256$, slice thickness $=1 \mathrm{~mm}$ and 8 accumulations). For in vitro imaging, the resonant circuit of the NMR probe was a 35-mm diameter birdcage resonator. PLA electropsun fibers were embedded in a degassed $1 \%(\mathrm{w} / \mathrm{w})$ agarose gel prior to imaging (Figure S11). Samples were analyzed using a two-dimensional acquisition with relaxation enhancement (RARE) sequence (repetition time $\mathrm{TR}=2500 \mathrm{~ms}$; effective echo time $\mathrm{TE}_{\mathrm{eff}}=33 \mathrm{~ms}$; RARE factor $=8$; FOV $=3 \mathrm{~cm} \times 2 \mathrm{~cm} ;$ matrix $128 \times 96$, slice thickness $=0.8 \mathrm{~mm}$ and 2 accumulations). Susceptibility weighted imaging (SWI) analyses were performed using a gradient echo sequence $(\mathrm{TR}=350 \mathrm{~ms}$; echo time $\mathrm{TE}=6 \mathrm{~ms}$; flip angle $=40^{\circ}, \mathrm{FOV}=3 \mathrm{~cm} \times 2 \mathrm{~cm}$; matrix $384 \times 384$, slice thickness $=0.5$ $\mathrm{mm})$ and presented as $\mathrm{T} 2 *$ weighted and filtered phase images.

Cytocompatibility. Cells and control polymer films were chosen in accordance with ISO 109935 guidelines. Mouse fibroblasts L929 cells (ECACC 85011425) were maintained in DMEM high 
glucose supplemented with 5\% Fetal Bovine Serum (FBS), 2mM L-glutamine and 1\% penicillin/streptomycin and cultured at $37^{\circ} \mathrm{C}$ and $5 \% \mathrm{CO}_{2}$. Cells were tested to be free of mycoplasms. Negative (RM-C High Density Polyethylene noted C-) and positive (RM-B 0.25\% ZDBC polyurethane noted $\mathrm{C}+$ ) control films were purchased from Hatano Research Institute (Ochiai 729-5, Hadanoshi, Kanagawa 257, Japan).

Cells proliferation on films. PLA films were cut to fit in wells of a 48-well plate. They were immerged for a few seconds in ethanol 70\%, rinsed with 3 baths of PBS-penicillin/streptomycin $10 \%$, and then with PBS only. L929 cells were seeded at $3.10^{4}$ cells per well in the center of a 48 well plate containing or not polymer films. After $1 \mathrm{~h}$ adhesion at room temperature, non-adherent cells were removed and, after a washing step, $500 \mu \mathrm{L}$ of growth medium was added. Cells were allowed to proliferate under appropriate atmosphere. Cell viability after $24 \mathrm{~h}, 48 \mathrm{~h}, 72 \mathrm{~h}$ and $144 \mathrm{~h}$ was assessed with Prestoblue ${ }^{\circledR}$ (Invitrogen), according to the manufacturer's protocol. Briefly, a mixture of $10 \%$ Prestoblue in growth medium was added to the cells, and the fluorescence at 590 $\mathrm{nm}$ was measured with CLARIOstar ${ }^{\circledR}$ (BMG LABTECH's) microplate reader after 45 minutes incubation $(\mathrm{n}=5)$.

Cytotoxicity assessed on extracts. First, extractions were carried out at $1 \mathrm{~cm}^{2}$ per $\mathrm{mL}$ for $72 \mathrm{~h}$ at $37^{\circ} \mathrm{C}$ under sterilized conditions on complete growth medium following ISO 10993-12 recommendations $(\mathrm{n}=3)$. L929 cells were seeded at $5.10^{3}$ cells per well in a 96-well plate and allowed to attach overnight. The culture medium was removed and discarded from the cultures and an aliquot of the extract $(100 \%)$ or dilution thereof $(75 \%, 50 \%, 25 \%)$ was added into each well. Aliquots of the blank, negative and positive controls were added into additional replicate wells $(\mathrm{n}=3)$. After $24 \mathrm{~h}$ incubation under appropriate atmosphere, extract's cytotoxicity was assessed by Lactate Dehydrogenase (LDH) assay (Pierce), according to the manufacturer's 
instruction. Briefly, medium from well was transferred to a new plate and mixed with LDH Reaction Mixture. After 30 minutes of incubation at room temperature, absorbance at $490 \mathrm{~nm}$ and $680 \mathrm{~nm}$ was measured using a CLARIOstar ${ }^{\circledR}$ microplate-reader (BMG LABTECH's).

Data Analysis. All biological data are expressed as means \pm SD and correspond to measurements in quintuplicate or triplicate. For cytocompatibility tests, Mann-Whitney was applied to determine the statistical significance (a level of $p<0.05$ was considered statistically significant).

\section{ASSOCIATED CONTENT}

\section{Supporting Information.}

The Supporting Information is available free of charge on the ACS Publications website, with the following information: Reaction conditions for the propargylation of PLA nanofibers; SEC curves of the pristine PLA and of the propargylated PLA; TEM image and TGA analysis (calculation of grafting density) of oleic acid functionalized SPIONs, Reaction conditions and results for strategy 1; SEM image at high magnification and TGA analysis of PLA@SPION for strategy 2; Displacement of PLA@SPIONs nanofibers supported on a glass slide (strategy 2) under the influence of a magnet and field dependence of the magnetization, and FC/ZFC curves for nongrafted SPION (PDF). Videos S1 and S2: Mobility and attraction of PLA@SPIONs fibers deposited under influence of magnet.

\section{AUTHOR INFORMATION}

\section{Corresponding Authors}


*Email: benjamin.nottelet@umontpellier.fr

*Email: danielle.laurencin@umontpellier.fr

\section{Author Contributions}

H. Awada and A. El Samad contributed equally to this work.

\section{Notes}

The authors declare no competing financial interest.

\section{ACKNOWLEDGEMENTS}

This work was partly funded by France Life Imaging (ANR-11-INBS-0006 grant from the French "Investissements d'Avenir" program) and was supported by the LabEx CheMISyst (ANR-10LABX-05-01 grant from the French "Investissements d'Avenir" program). It was also supported by the Azm \& Saade Association of Lebanon (A.A.S), the Lebanese Association for Scientific Research (LASeR) (A.E.J), and a Partner University Fund between the University of Montpellier and the Rensselaer Polytechnic Institute (D. L., R. G. \& R. P.). The authors also thank the University of Montpellier and CNRS for financial support. We thank C. Rebeil (PAC ICGM) for magnetic measurements and B. Rebière for SEM and EDXS analysis.

\section{REFERENCES}

1. Wahajuddin; Arora, S., Superparamagnetic Iron Oxide Nanoparticles: Magnetic Nanoplatforms as Drug Carriers. Int. J. Nanomed. 2012, 7, 3445-3471. 
2. Bao, Y.; Sherwood, J. A.; Sun, Z., Magnetic Iron Oxide Nanoparticles as T1 Contrast Agents for Magnetic Resonance Imaging. J. Mater. Chem. C 2018, 6 (6), 1280-1290.

3. Lee, S. J.; Muthiah, M.; Lee, H. J.; Lee, H. J.; Moon, M. J.; Che, H. L.; Heo, S. U.; Lee, H. C.; Jeong, Y. Y.; Park, I. K., Synthesis and Characterization of Magnetic Nanoparticle-Embedded Multi-Functional Polymeric Micelles for Mri-Guided Gene Delivery. Macromol. Res. 2012, 20 (2), 188-196.

4. Stephen, Z. R.; Dayringer, C. J.; Lim, J. J.; Revia, R. A.; Halbert, M. V.; Jeon, M.; Bakthavatsalam, A.; Ellenbogen, R. G.; Zhang, M. Q., Approach to Rapid Synthesis and Functionalization of Iron Oxide Nanoparticles for High Gene Transfection. ACS Appl. Mater. Interfaces 2016, 8 (10), 6320-6328.

5. Thomas, R. G.; Moon, M. J.; Lee, H.; Sasikala, A. R. K.; Kim, C. S.; Park, I. K.; Jeong, Y. Y., Hyaluronic Acid Conjugated Superparamagnetic Iron Oxide Nanoparticle for Cancer Diagnosis and Hyperthermia Therapy. Carbohydr. Polym. 2015, 131, 439-446.

6. Connell, J. J.; Patrick, P. S.; Yu, Y. C.; Lythgoe, M. F.; Kalber, T. L., Advanced Cell Therapies: Targeting, Tracking and Actuation of Cells with Magnetic Particles. Regen. Med. 2015, $10(6), 757-772$.

7. Lewin, M.; Carlesso, N.; Tung, C. H.; Tang, X. W.; Cory, D.; Scadden, D. T.; Weissleder, R., Tat Peptide-Derivatized Magnetic Nanoparticles Allow in Vivo Tracking and Recovery of Progenitor Cells. Nat. Biotechnol. 2000, 18 (4), 410-414.

8. Marie, H.; Lemaire, L.; Franconi, F.; Lajnef, S.; Frapart, Y. M.; Nicolas, V.; Frebourg, G.; Trichet, M.; Menager, C.; Lesieur, S., Superparamagnetic Liposomes for Mri Monitoring and External Magnetic Field-Induced Selective Targeting of Malignant Brain Tumors. Adv. Funct. Mater. 2015, 25 (8), 1258-1269.

9. $\quad$ Park, K. S.; Kim, H.; Kim, S.; Lee, K.; Park, S.; Song, J.; Min, C.; Khanam, F.; Rashu, R.; Bhuiyan, T. R.; Ryan, E. T.; Qadri, F.; Weissleder, R.; Cheon, J.; Charles, R. C.; Lee, H., Nanomagnetic System for Rapid Diagnosis of Acute Infection. Acs Nano 2017, 11 (11), 1142511432.

10. Thapa, B.; Diaz-Diestra, D.; Santiago-Medina, C.; Kumar, N.; Tu, K.; Beltran-Huarac, J.; Jadwisienczak, W. M.; Weiner, B. R.; Morell, G., T1- and T2-Weighted Magnetic Resonance Dual Contrast by Single Core Truncated Cubic Iron Oxide Nanoparticles with Abrupt Cellular Internalization and Immune Evasion. ACS Appl. Bio Mater. 2018, 1 (1), 79-89. 
11. Chapon, C.; Franconi, F.; Lacoeuille, F.; Hindre, F.; Saulnier, P.; Benoit, J. P.; Le Jeune, J. J.; Lemaire, L., Imaging E-Selectin Expression Following Traumatic Brain Injury in the Rat Using a Targeted Uspio Contrast Agent. Magn. Reson. Mater. Phy. 2009, 22 (3), 167-174.

12. Montet, X.; Montet-Abou, K.; Reynolds, F.; Weissleder, R.; Josephson, L., Nanoparticle Imaging of Integrins on Tumor Cells. Neoplasia 2006, 8 (3), 214-222.

13. Pinney, J. R.; Melkus, G.; Cerchiari, A.; Hawkins, J.; Desai, T. A., Novel Functionalization of Discrete Polymeric Biomaterial Microstructures for Applications in Imaging and ThreeDimensional Manipulation. ACS Appl. Mater. Interfaces 2014, 6 (16), 14477-14485.

14. Sivaraman, B.; Swaminathan, G.; Moore, L.; Fox, J.; Seshadri, D.; Dahal, S.; Stoilov, I.; Zborowski, M.; Mecham, R.; Ramamurthi, A., Magnetically-Responsive, Multifunctional Drug Delivery Nanoparticles for Elastic Matrix Regenerative Repair. Acta Biomater. 2017, 52 (1), 171186.

15. Jin, R. R.; Lin, B. B.; Li, D. Y.; Ai, H., Superparamagnetic Iron Oxide Nanoparticles for Mr Imaging and Therapy: Design Considerations and Clinical Applications. Curr. Opin. Pharmacol. 2014, 18, 18-27.

16. Thomas, R.; Park, I. K.; Jeong, Y. Y., Magnetic Iron Oxide Nanoparticles for Multimodal Imaging and Therapy of Cancer. Int. J. Mol. Sci. 2013, 14 (8), 15910-15930.

17. Jeong, Y.; Hwang, H. S.; Na, K., Theranostics and Contrast Agents for Magnetic Resonance Imaging. Biomater. Res. 2018, 22 (1), 20.

18. Valdiglesias, V.; Fernandez-Bertolez, N.; Kilic, G.; Costa, C.; Costa, S.; Fraga, S.; Bessa, M. J.; Pasaro, E.; Teixeira, J. P.; Laffon, B., Are Iron Oxide Nanoparticles Safe? Current Knowledge and Future Perspectives. J. Trace Elem. Med Biol. 2016, 38, 53-63.

19. Amstad, E.; Textor, M.; Reimhult, E., Stabilization and Functionalization of Iron Oxide Nanoparticles for Biomedical Applications. Nanoscale 2011, 3 (7), 2819-2843.

20. Boyer, C.; Whittaker, M. R.; Bulmus, V.; Liu, J. Q.; Davis, T. P., The Design and Utility of Polymer-Stabilized Iron-Oxide Nanoparticles for Nanomedicine Applications. Npg Asia Mater 2010, 2 (1), 23-30.

21. Muthiah, M.; Park, I. K.; Cho, C. S., Surface Modification of Iron Oxide Nanoparticles by Biocompatible Polymers for Tissue Imaging and Targeting. Biotechnol. Adv. 2013, 31 (8), 12241236. 
22. Unterweger, H.; Janko, C.; Schwarz, M.; Dezsi, L.; Urbanics, R.; Matuszak, J.; Orfi, E.; Fulop, T.; Bauerle, T.; Szebeni, J.; Journe, C.; Boccaccini, A. R.; Alexiou, C.; Lyer, S.; Cicha, I., Non-Immunogenic Dextran-Coated Superparamagnetic Iron Oxide Nanoparticles: A Biocompatible, Size-Tunable Contrast Agent for Magnetic Resonance Imaging. Int. J. Nanomed. 2017, 12, 5223-5238.

23. Weissleder, R.; Stark, D. D.; Engelstad, B. L.; Bacon, B. R.; Compton, C. C.; White, D. L.; Jacobs, P.; Lewis, J., Superparamagnetic Iron-Oxide - Pharmacokinetics and Toxicity. Am. J. Roentgenol. 1989, 152 (1), 167-173.

24. Szpak, A.; Kania, G.; Skorka, T.; Tokarz, W.; Zapotoczny, S.; Nowakowska, M., Stable Aqueous Dispersion of Superparamagnetic Iron Oxide Nanoparticles Protected by Charged Chitosan Derivatives. J. Nanopart. Res. 2013, 15 (1), 1372.

25. Gautier, J.; Munnier, E.; Paillard, A.; Herve, K.; Douziech-Eyrolles, L.; Souce, M.; Dubois, P.; Chourpa, I., A Pharmaceutical Study of Doxorubicin-Loaded Pegylated Nanoparticles for Magnetic Drug Targeting. Int. J. Pharm. 2012, 423 (1), 16-25.

26. Prabhu, S.; Mutalik, S.; Rai, S.; Udupa, N.; Rao, B. S. S., Pegylation of Superparamagnetic Iron Oxide Nanoparticle for Drug Delivery Applications with Decreased Toxicity: An in Vivo Study. J. Nanopart. Res. 2015, 17 (10), 412.

27. Thapa, B.; Diaz-Diestra, D.; Beltran-Huarac, J.; Weiner, B. R.; Morell, G., Enhanced Mri T2 Relaxivity in Contrast-Probed Anchor-Free Pegylated Iron Oxide Nanoparticles. Nanoscale Res. Lett. 2017, 12 (1), 312.

28. Kurtan, U.; Esir, S.; Baykal, A.; Sozeri, H., Poly(Amidoamine)-Grafted Superparamagnetic Iron Oxide Nanoparticles: Synthesis and Characterization. J. Supercond. Nov. Magn. 2014, 27 (9), 2097-2103.

29. Zhao, L.; Chano, T.; Morikawa, S.; Saito, Y.; Shiino, A.; Shimizu, S.; Maeda, T.; Irie, T.; Aonuma, S.; Okabe, H.; Kimura, T.; Inubushi, T.; Komatsu, N., Hyperbranched PolyglycerolGrafted Superparamagnetic Iron Oxide Nanoparticles: Synthesis, Characterization, Functionalization, Size Separation, Magnetic Properties, and Biological Applications. Adv. Funct. Mater. 2012, 22 (24), 5107-5117.

30. Kurzhals, S.; Gal, N.; Zirbs, R.; Reimhult, E., Controlled Aggregation and Cell Uptake of Thermoresponsive Polyoxazoline-Grafted Superparamagnetic Iron Oxide Nanoparticles. Nanoscale 2017, 9 (8), 2793-2805. 
31. Huang, S. J.; Ke, J. H.; Chen, G. J.; Wang, L. F., One-Pot Synthesis of Pdmaema-Bound Iron Oxide Nanoparticles for Magnetofection. J. Mater. Chem. B. 2013, 1 (43), 5916-5924.

32. Kurzhals, S.; Pretzner, B.; Reimhult, E.; Zirbs, R., Thermoresponsive Polypeptoid-Coated Superparamagnetic Iron Oxide Nanoparticles by Surface-Initiated Polymerization. Macromol. Chem. Phys. 2017, 218 (13), 1700116.

33. Sulek, S.; Mammadov, B.; Mahcicek, D. I.; Sozeri, H.; Atalar, E.; Tekinay, A. B.; Guler, M. O., Peptide Functionalized Superparamagnetic Iron Oxide Nanoparticles as Mri Contrast Agents. J. Mater. Chem. 2011, 21 (39), 15157-15162.

34. Ulbrich, K.; Hola, K.; Subr, V.; Bakandritsos, A.; Tucek, J.; Zboril, R., Targeted Drug Delivery with Polymers and Magnetic Nanoparticles: Covalent and Noncovalent Approaches, Release Control, and Clinical Studies. Chem. Rev. 2016, 116 (9), 5338-5431.

35. Zuidema, J. M.; Provenza, C.; Caliendo, T.; Dutz, S.; Gilbert, R. J., Magnetic NgfReleasing Plla/Iron Oxide Nanoparticles Direct Extending Neurites and Preferentially Guide Neurites Along Aligned Electrospun Microfibers. Acs Chem. Neurosci. 2015, 6 (11), 1781-1788.

36. Nottelet, B.; Darcos, V.; Coudane, J., Aliphatic Polyesters for Medical Imaging and Theranostic Applications. Eur. J. Pharm. Biopharm. 2015, 97, 350-370.

37. Ai, H.; Flask, C.; Weinberg, B.; Shuai, X.; Pagel, M. D.; Farrell, D.; Duerk, J.; Gao, J. M., Magnetite-Loaded Polymeric Micelles as Ultrasensitive Magnetic-Resonance Probes. Adv. Mater. 2005, 17 (16), 1949-1952.

38. Kim, H. C.; Kim, E.; Jeong, S. W.; Ha, T. L.; Park, S. I.; Lee, S. G.; Lee, S. J.; Lee, S. W., Magnetic Nanoparticle-Conjugated Polymeric Micelles for Combined Hyperthermia and Chemotherapy. Nanoscale 2015, 7 (39), 16470-16480.

39. Prashant, C.; Dipak, M.; Yang, C. T.; Chuang, K. H.; Jun, D.; Feng, S. S., Superparamagnetic Iron Oxide - Loaded Poly (Lactic Acid)-D-Alpha-Tocopherol Polyethylene Glycol 1000 Succinate Copolymer Nanoparticles as Mri Contrast Agent. Biomaterials 2010, 31 (21), 5588-5597.

40. Tang, Z. M.; Zhang, L.; Wang, Y.; Li, D.; Zhong, Z. D.; Zhou, S. B., Redox-Responsive Star-Shaped Magnetic Micelles with Active-Targeted and Magnetic-Guided Functions for Cancer Therapy. Acta Biomater. 2016, 42, 232-246.

41. Zou, H.; Yuan, W. Z., Temperature- and Redox-Responsive Magnetic Complex Micelles for Controlled Drug Release. J. Mater. Chem. B. 2015, 3 (2), 260-269. 
42. Dadras, P.; Atyabi, F.; Irani, S.; Ma'mani, L.; Foroumadi, A.; Mirzaie, Z. H.; Ebrahimi, M.; Dinarvand, R., Formulation and Evaluation of Targeted Nanoparticles for Breast Cancer Theranostic System. Eur. J. Pharm. Sci. 2017, 97, 47-54.

43. Shanavas, A.; Sasidharan, S.; Bahadur, D.; Srivastava, R., Magnetic Core-Shell Hybrid Nanoparticles for Receptor Targeted Anti-Cancer Therapy and Magnetic Resonance Imaging. $J$. Colloid. Interf. Sci. 2017, 486, 112-120.

44. Geilich, B. M.; Gelfat, I.; Sridhar, S.; van de Ven, A. L.; Webster, T. J., Superparamagnetic Iron Oxide-Encapsulating Polymersome Nanocarriers for Biofilm Eradication. Biomaterials 2017, $119,78-85$.

45. Chang, W. J.; Pan, Y. H.; Tzeng, J. J.; Wu, T. L.; Fong, T. H.; Feng, S. W.; Huang, H. M., Development and Testing of X-Ray Imaging-Enhanced Poly-L-Lactide Bone Screws. Plos One 2015, 10 (10), e0140354.

46. Sarkar, S.; Guibal, E.; Quignard, F.; SenGupta, A. K., Polymer-Supported Metals and Metal Oxide Nanoparticles: Synthesis, Characterization, and Applications. J. Nanopart. Res. 2012, $14(2), 715$.

47. Campbell, S. B.; Patenaude, M.; Hoare, T., Injectable Superparamagnets: Highly Elastic and Degradable Poly(N-Isopropylacrylamide)-Superparamagnetic Iron Oxide Nanoparticle (Spion) Composite Hydrogels. Biomacromolecules 2013, 14 (3), 644-653.

48. Fu, J. C.; Wang, Q. F.; Schlenoff, J. B., Extruded Superparamagnetic Saloplastic Polyelectrolyte Nanocomposites. ACS Appl. Mater. Interfaces 2015, 7 (1), 895-901.

49. Lee, H. J.; Lee, S. J.; Uthaman, S.; Thomas, R. G.; Hyun, H.; Jeong, Y. Y.; Cho, C. S.; Park, I. K., Biomedical Applications of Magnetically Functionalized Organic/Inorganic Hybrid Nanofibers. Int. J. Mol. Sci. 2015, 16 (6), 13661-13677.

50. Mortimer, C. J.; Wright, C. J., The Fabrication of Iron Oxide Nanoparticle-Nanofiber Composites by Electrospinning and Their Applications in Tissue Engineering. Biotechnol. J. 2017, $12(7), 1600693$.

51. Meng, J.; Xiao, B.; Zhang, Y.; Liu, J.; Xue, H. D.; Lei, J.; Kong, H.; Huang, Y. G.; Jin, Z. Y.; Gu, N.; Xu, H. Y., Super-Paramagnetic Responsive Nanofibrous Scaffolds under Static Magnetic Field Enhance Osteogenesis for Bone Repair in Vivo. Sci Rep-Uk 2013, 3, 2655. 
52. Meng, J.; Zhang, Y.; Qi, X. J.; Kong, H.; Wang, C. Y.; Xu, Z.; Xie, S. S.; Gu, N.; Xu, H. Y., Paramagnetic Nanofibrous Composite Films Enhance the Osteogenic Responses of PreOsteoblast Cells. Nanoscale 2010, 2 (12), 2565-2569.

53. Shan, D. Y.; Shi, Y. Z.; Duan, S.; Wei, Y.; Cai, Q.; Yang, X. P., Electrospun Magnetic Poly(L-Lactide) (Plla) Nanofibers by Incorporating Plla-Stabilized Fe3o4 Nanoparticles. Mat. Sci. Eng. C-Mater. 2013, 33 (6), 3498-3505.

54. Singh, R. K.; Patel, K. D.; Lee, J. H.; Lee, E. J.; Kim, J. H.; Kim, T. H.; Kim, H. W., Potential of Magnetic Nanofiber Scaffolds with Mechanical and Biological Properties Applicable for Bone Regeneration. Plos One 2014, 9 (4), 0091584.

55. Echeverry-Rendon, M.; Reece, L. M.; Pastrana, F.; Arias, S. L.; Shetty, A. R.; Pavon, J. J.; Allain, J. P., Bacterial Nanocellulose Magnetically Functionalized for Neuro-Endovascular Treatment. Macromol. Biosci. 2017, 17 (6), 1600382.

56. Brismar, T. B.; Grishenkov, D.; Gustafsson, B.; Harmark, J.; Barrefelt, A.; Kothapalli, S. V. V. N.; Margheritelli, S.; Oddo, L.; Caidahl, K.; Hebert, H.; Paradossi, G., Magnetite Nanoparticles Can Be Coupled to Microbubbles to Support Multimodal Imaging. Biomacromolecules 2012, 13 (5), 1390-1399.

57. He, W.; Yang, F.; Wu, Y. H.; Wen, S.; Chen, P.; Zhang, Y.; Gu, N., Microbubbles with Surface Coated by Superparamagnetic Iron Oxide Nanoparticles. Mater. Lett. 2012, 68, 64-67.

58. Sciallero, C.; Balbi, L.; Paradossi, G.; Trucco, A., Magnetic Resonance and Ultrasound Contrast Imaging of Polymer-Shelled Microbubbles Loaded with Iron Oxide Nanoparticles. Roy. Soc. Open Sci. 2016, 3 (8), 160063.

59. Kuo, C. Y.; Liu, T. Y.; Hardiansyah, A.; Chiu, W. Y., Magnetically Polymeric Nanocarriers for Targeting Delivery of Curcumin and Hyperthermia Treatments toward Cancer Cells. J. Polym. Sci. Pol. Chem. 2016, 54 (17), 2706-2713.

60. El Habnouni, S.; Darcos, V.; Garric, X.; Lavigne, J. P.; Nottelet, B.; Coudane, J., Mild Methodology for the Versatile Chemical Modification of Polylactide Surfaces: Original Combination of Anionic and Click Chemistry for Biomedical Applications. Adv. Funct. Mater. 2011, 21 (17), 3321-3330.

61. Sardo, C.; Nottelet, B.; Triolo, D.; Giammona, G.; Garric, X.; Lavigne, J. P.; Cavallaro, G.; Coudane, J., When Functionalization of Pla Surfaces Meets Thiol-Yne Photochemistry: Case 
Study with Antibacterial Polyaspartamide Derivatives. Biomacromolecules 2014, 15 (11), 43514362.

62. Guerrero, G.; Alauzun, J. G.; Granier, M.; Laurencin, D.; Mutin, P. H., Phosphonate Coupling Molecules for the Control of Surface/Interface Properties and the Synthesis of Nanomaterials. Dalton Trans. 2013, 42 (35), 12569-12585.

63. Li, L.; Feng, W. Q.; Welle, A.; Levkin, P. A., Uv-Induced Disulfide Formation and Reduction for Dynamic Photopatterning. Angew. Chem. Int. Edit. 2016, 55 (44), 13765-13769.

64. Lartigue, L.; Innocenti, C.; Kalaivani, T.; Awwad, A.; Sanchez Duque, M. d. M.; Guari, Y.; Larionova, J.; Guérin, C.; Montero, J.-L. G.; Barragan-Montero, V.; Arosio, P.; Lascialfari, A.; Gatteschi, D.; Sangregorio, C., Water-Dispersible Sugar-Coated Iron Oxide Nanoparticles. An Evaluation of Their Relaxometric and Magnetic Hyperthermia Properties. J. Am. Chem. Soc. 2011, 133 (27), 10459-10472.

65. Chang, K. C.; Su, I. H.; Senthilvelan, A.; Chung, W. S., Triazole-Modified Calix[4]Crown as a Novel Fluorescent on-Off Switchable Chemosensor. Org. Lett. 2007, 9 (17), 3363-3366.

66. Sun, X.; Frey Huls, N.; Sigdel, A.; Sun, S., Tuning Exchange Bias in Core/Shell Feo/Fe3o4 Nanoparticles. Nano Letters 2012, 12 (1), 246-251.

67. Johnson, C. D. L.; D'Amato, A. R.; Gilbert, R. J., Electrospun Fibers for Drug Delivery after Spinal Cord Injury and the Effects of Drug Incorporation on Fiber Properties. Cells Tissues Organs 2015, 202 (1-2), 116-135.

68. Sene, S.; McLane, J.; Schaub, N.; Begu, S.; Mutin, P. H.; Ligon, L.; Gilbert, R. J.; Laurencin, D., Formulation of Benzoxaborole Drugs in Plla: From Materials Preparation to in Vitro Release Kinetics and Cellular Assays. J. Mater. Chem. B. 2016, 4 (2), 257-272.

69. Yu, W. W.; Falkner, J. C.; Yavuz, C. T.; Colvin, V. L., Synthesis of Monodisperse Iron Oxide Nanocrystals by Thermal Decomposition of Iron Carboxylate Salts. Chem. Commun. 2004, (20), 2306-2307.

70. Harris, E. S.; Morgan, R. F.; Rodeheaver, G. T., Analysis of the Kinetics of Peritoneal Adhesion Formation in the Rat and Evaluation of Potential Antiadhesive Agents. Surgery 1995, 117 (6), 663-669. 


\section{GRAPHICAL TABLE OF CONTENTS}

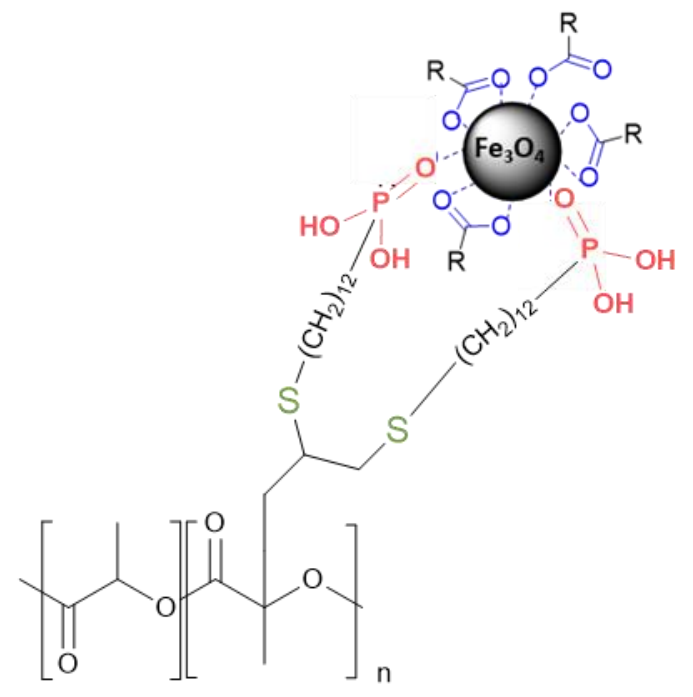

PLA nanofibers

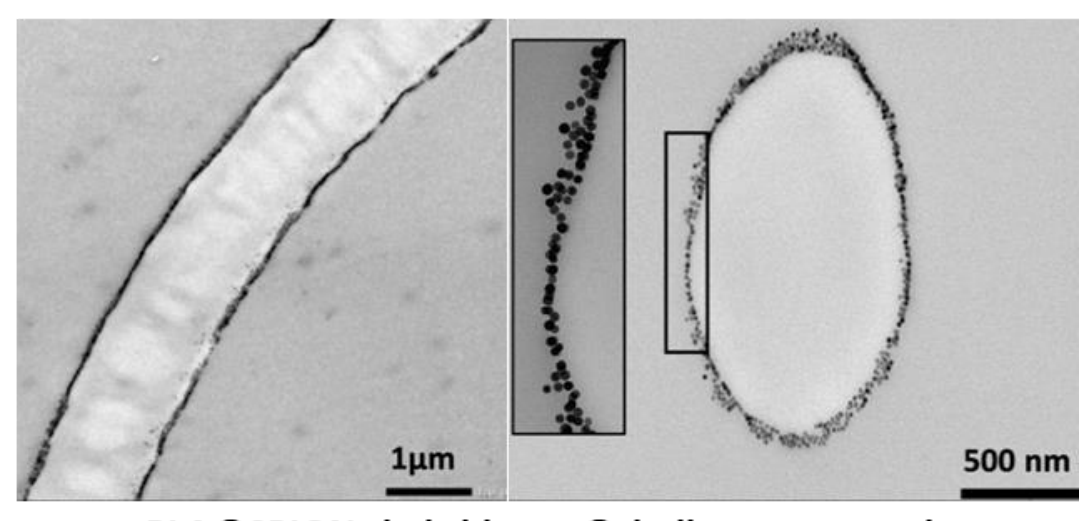

PLA@SPIONs hybrid core@shell nanocomposites 